\title{
COMPUTING DISCRETE LOGARITHMS IN THE JACOBIAN OF HIGH-GENUS HYPERELLIPTIC CURVES OVER EVEN CHARACTERISTIC FINITE FIELDS
}

\author{
M. D. VELICHKA, M. J. JACOBSON JR., AND A. STEIN
}

\begin{abstract}
We describe improved versions of index-calculus algorithms for solving discrete logarithm problems in Jacobians of high-genus hyperelliptic curves defined over even characteristic fields. Our first improvement is to incorporate several ideas for the low-genus case by Gaudry and Thériault, including the large prime variant and using a smaller factor base, into the large-genus algorithm of Enge and Gaudry. We extend the analysis in a 2001 paper by Jacobson, Menzes, and Stein to our new algorithm, allowing us to predict accurately the number of random walk steps required to find all relations, and to select optimal degree bounds for the factor base. Our second improvement is the adaptation of sieving techniques from Flassenberg and Paulus, and Jacobson to our setting. The new algorithms are applied to concrete problem instances arising from the Weil descent attack methodology for solving the elliptic curve discrete logarithm problem, demonstrating significant improvements in practice.
\end{abstract}

\section{INTRODUCTION}

Elliptic curves [28, 34] and hyperelliptic curves [29] were proposed for use in public-key cryptographic protocols based on the discrete logarithm problem in the Jacobian of these curves. Elliptic curves have become a popular choice in many protocols. Recent attacks (see, e.g., [16, 17, 39]) show that hyperelliptic curves of genus 2 and possibly genus 3 have comparable properties.

Hyperelliptic curves are not only of interest on the constructive side. High genus hyperelliptic curves are of cryptographic interest in the analysis of elliptic curve cryptosystems in the context of the Weil descent attack methodology [13,15. Weil descent allows one to reduce the elliptic curve discrete logarithm problem (ECDLP) on some elliptic curves defined over a finite field of composite degree to an instance of the hyperelliptic curve discrete problem (HCDLP) of high genus defined over a smaller field. Under certain circumstances, the resulting instance of the HCDLP can be solved in subexponential time using index calculus algorithms. In our case, we only consider fields of characteristic 2 .

In [25, the authors looked at the cost of using Weil descent to solve some specific instances of the elliptic curve discrete logarithm problem in practice. The main tool was an improved version of the Enge-Gaudry algorithm [10] for solving instances

Received by the editor February 7, 2011 and, in revised form, July 3, 2012.

2010 Mathematics Subject Classification. Primary 14G50; Secondary 11G20, 11Y16, 11 Y40.

Key words and phrases. Hyperelliptic curves, discrete logarithm problem, sieving, Weil descent.

The first author's research was supported by NSERC of Canada.

The second author's research was supported by NSERC of Canada.

(C)2013 American Mathematical Society Reverts to public domain 28 years from publication 
of the hyperelliptic curve discrete logarithm problem over high-genus curves, which includes an enhanced smoothness-testing algorithm and a strategy for selecting an optimal factor base empirically. Four instances of the elliptic curve discrete logarithm problem were considered for which the curves were defined over $\mathbb{F}_{2^{62}}$, $\mathbb{F}_{2^{93}}, \mathbb{F}_{2^{124}}$, and $\mathbb{F}_{2^{155}}$. Weil descent reduces these to instances of the HCDLP where the curves have genus 31 and are defined over $\mathbb{F}_{2^{2}}, \mathbb{F}_{2^{3}}, \mathbb{F}_{2^{4}}$, and $\mathbb{F}_{2^{5}}$. The first three discrete logarithm problems were solved using a parallel implementation of the improved Enge-Gaudry algorithm, and estimates for the fourth example were given indicating that it should be solvable, as the time required is comparable to that required to break the data encryption standard block cipher.

In this paper, 1 we describe further practical improvements to the Enge-Gaudry algorithm in the large genus case that allowed us to solve the remaining discrete logarithm problem from [25] (elliptic curve over $\mathbb{F}_{2^{155}}$ ) in less time than predicted. The algorithm is extended, in terms of both the implementation and the empirical parameter selection strategy, to incorporate the large prime variants described by Thériault [39] for low-genus hyperelliptic curves. In addition, the sieving strategy for relation generation of Flassenberg and Paulus [11] is optimized for characteristic 2 and extended to work with the well-known self-initialization strategy employed in integer factorization and index-calculus in quadratic number fields. The resulting algorithms are applied to the same Weil descent examples as in [25], showing that each of the modifications does indeed lead to improved performance in practice.

This paper is organized as follows. We describe the relevant background material on hyperelliptic curves and the hyperelliptic curve discrete logarithm problem in Section 2. Our adaptation of Thériault's large prime variation [39] to the version of the Enge-Gaudry algorithm from [25], as well as the revised empirical estimates for parameter selection, are described in Section 3 The sieve-based improvements are described in Section 4. Our numerical results are presented in Section 5. followed by a summary of possible future research directions.

\section{HyPERELLIPTIC CURVES}

For details on hyperelliptic curves arithmetic, we refer to [5, 7, 27. Here, we briefly sketch the basics.

Let $k=\mathbb{F}_{q}$ denote the finite field with $q$ elements and $\bar{k}=\bigcup_{n>1} \mathbb{F}_{q^{n}}$ its algebraic closure. A hyperelliptic curve $C$ of genus $g$ over $k$ is defined by a non-singular equation

$$
v^{2}+h(u) v=f(u),
$$

where $h, f \in k[u], \operatorname{deg} f=2 g+1$, and $\operatorname{deg} h \leq g$. Let $L$ be an extension field of $k$. The set of points on $C$ is $C(\bar{k})=\left\{(x, y): x, y \in \bar{k}, y^{2}+h(x) y=f(x)\right\} \cup\{\infty\}$. The opposite of $P=(x, y) \in C(\bar{k}) \backslash\{\infty\}$ is $-P=(x,-y-h(x))$ and $-\infty=\infty$.

2.1. Jacobian of a hyperelliptic curve. A degree zero divisor $D$ of $C$ is a formal sum $\sum_{P \in C(\bar{k})} m_{P} P$, where $m_{P} \in \mathbb{Z}$, only a finite number of the $m_{P}$ 's are non-zero, and $\sum m_{P}=0$. The set $D^{0}$ of zero divisors is an additive group under formal addition $\sum m_{P} P+\sum n_{P} P=\sum\left(m_{P}+n_{P}\right) P$. The Frobenius map $\sigma: \bar{k} \rightarrow \bar{k}, x \mapsto x^{q}$ extends naturally to $C(\bar{k})$ by $(x, y) \mapsto\left(x^{\sigma}, y^{\sigma}\right)$ and $\infty^{\sigma} \mapsto \infty$, and homomorphically to $D^{0}$ by $\sum m_{P} P \mapsto \sum m_{P} P^{\sigma}$. Let $D_{k}^{0}=\left\{D \in D^{0}: D^{\sigma}=D\right\}$ be the set of zero

\footnotetext{
40.

${ }^{1}$ The results presented in this paper are described in more detail in M. Velichka's M.Sc. thesis
} 
divisors defined over $k$, i.e., fixed by the Frobenius map, and let $k(C)$ be the function field of $C$ over $k$, i.e., the field of fractions of the integral domain of polynomial functions $k[u, v] /\left(v^{2}+h(u) v-f(u)\right)$. For $f \in k(C)$, the divisor of $f$ is defined as $\operatorname{div}(f)=\sum_{P \in C(\bar{k})} v_{P}(f) P$, where $v_{P}(f)$ denotes the multiplicity of $P$ as a root of $f$. If we let $\operatorname{Prin}_{k}=\{\operatorname{div}(f): f \in k(C)\}$, then $\operatorname{Prin}_{k}$ is a subgroup of $D_{k}^{0}$. Finally, the Jacobian of $C$ defined over $k$ is the quotient group $J_{C}(k)=D_{k}^{0} / \operatorname{Prin}_{k}$.

The Jacobian $J_{C}(k)$ is a finite abelian group of size $\# J_{C}(k) \approx q^{g}$. We write $D_{1} \sim D_{2}$ to denote that $D_{1}$ and $D_{2}$ lie in the same equivalence class of divisors in $J_{C}(k)$. In our situation, we know that each equivalence class has a unique reduced divisor, i.e., a divisor $\sum_{P \neq \infty} m_{P} P-\left(\sum_{P \neq \infty} m_{P}\right) \infty$ satisfying (i) $m_{P} \geq 0$ for all $P$; (ii) if $m_{P} \geq 1$ and $P \neq-P$, then $m_{-P}=0$; (iii) $m_{P}=0$ or 1 if $P=-P$; and (iv) $\sum m_{P} \leq g$. Each reduced divisor can be uniquely represented by a pair of polynomials $a, b \in k[u]$ where (i) $\operatorname{deg} b<\operatorname{deg} a \leq g$; (ii) $a$ is monic; and (iii) $a \mid\left(b^{2}+b h-f\right)$. We write $D=\operatorname{div}(a, b)$ to mean $D=\operatorname{gcd}(\operatorname{div}(a), \operatorname{div}(b-v))$ where the gcd of two divisors $\sum m_{P} P$ and $\sum n_{P} P$ is defined to be $\sum \min \left(m_{P}, n_{P}\right) P$. The identity of $J_{C}(k)$ is represented by the the divisor $\operatorname{div}(1,0)$.

In order to measure the size of a divisor, we now define the degree of $D=\operatorname{div}(a, b)$ as $\operatorname{deg} D=\operatorname{deg} a$. Notice that by the degree of a divisor, we do not mean the degree of $D \in D^{0}$ as a formal sum (which is of course 0 ). Similarly, we define the norm of $D$ to be $N(D)=a$, and note that $N\left(D_{1}+D_{2}\right)=N\left(D_{1}\right) N\left(D_{2}\right)$, where $D_{1}+D_{2}$ denotes the formal sum.

The group law in $J_{C}(k)$ can be efficiently computed via NUCOMP as described in [26]. For two reduced divisors $D_{1}$ and $D_{2}$, a reduced divisor $D_{3} \sim D_{1}+D_{2}$ can be determined by this algorithm in polynomial time.

2.2. The hyperelliptic curve discrete logarithm problem. Let $C$ be a genus $g$ hyperelliptic curve over $k=\mathbb{F}_{q}$. The hyperelliptic curve discrete logarithm problem (HCDLP) is the following: given $C, D_{1} \in J_{C}(k), r=\operatorname{ord}\left(D_{1}\right)$, and $D_{2} \in\left\langle D_{1}\right\rangle$, find the integer $s \in[0, r-1]$ such that $D_{2}=s D_{1}$. We shall assume that $r$ is prime, and $\# J_{C}(k) / r$ is small.

Enge and Gaudry [10] developed an index-calculus algorithm suitable for solving the HCDLP on high-genus hyperelliptic curves. Under the assumptions that $g / \log q \rightarrow \infty$ and that $\# J_{C}(k)$ is known, their algorithm requires $L_{q^{g}}[\sqrt{2}]=$ $L_{q^{2 g+1}}[1]$ bit operations, where $L_{N}[\alpha]=\exp ((\alpha+o(1)) \sqrt{\log N \log \log N})$. Our improved algorithms are based on the Enge-Gaudry method; we begin with an overview of this algorithm.

The key to the efficiency of any index-calculus algorithm, including the EngeGaudry algorithm, is being able to efficiently and with high probability find and recognize elements that are "smooth" in some sense. In the hyperelliptic curve case, we need to find divisors that are the sum of small-degree prime divisors. A reduced divisor $D=\operatorname{div}(a, b) \in J_{C}(k)$ is called a prime divisor if $a$ is irreducible over $k$. Throughout the paper, primes and prime divisors are simply identified with reduced divisors $\operatorname{div}(p, b) \in J_{C}(k)$, denoted as $P=\operatorname{div}(p, b)$, where $p$ is a prime polynomial. Its degree is $\operatorname{deg} P=\operatorname{deg} p$. The size of $P$ is measured by the degree of $p$ as a polynomial (even though its degree as formal divisors is 0 ). Therefore, it makes sense to define smooth divisors as follows: A reduced divisor is said to be $t$-smooth if it can be expressed as a sum of prime divisors that all have degree $\leq t$.

The set of all prime divisors of degree $\leq t$ can be found as follows. For each monic irreducible polynomial $p \in k[u]$ of degree $\leq t$, find the roots of $v^{2}+h(u) v-f(u)$ 
modulo $p(u)$. For each root $b(u)$ (there are either 0,1 or 2 such $\operatorname{roots}), \operatorname{div}(p, b)$ is a prime divisor. In order to ensure we always use the same prime divisor for a given polynomial $p$ in the cases where 2 roots exist, we take the root $b(u)$ for which the integer value of $b(u)$ evaluated at $q$ is smaller.

A reduced divisor $D=\operatorname{div}(a, b) \in J_{C}(k)$ can be efficiently decomposed into prime divisors in an expected time polynomial in the degree of $a$ and $b$. Simply factor $a$ into monic irreducibles over $k$ and obtain $a=\prod_{i=1}^{L} a_{i}^{e_{i}}$. Then $D=\sum_{i=1}^{L} e_{i} \operatorname{div}\left(a_{i}, b_{i}\right)$, where $b_{i}=b \bmod a_{i}$ for $1 \leq i \leq L$.

2.3. The Enge-Gaudry index-calculus algorithm. As in any index-calculus algorithm, the Enge-Gaudry index-calculus algorithm requires us to choose a factor base $S$ of suitable prime elements of small norm. Select a degree bound $t$ and define the factor base $S=\left\{P_{1}, P_{2}, \ldots, P_{w}\right\}$ as the set of prime divisors of degree $\leq t$. The crucial input is a pseudo-random walk (see, e.g., 38]) in the set of reduced divisors. This walk produces random linear combinations of the form $\alpha D_{1}+\beta D_{2}$. If the reduced divisor $R$ equivalent to this form is $t$-smooth, it will be stored. This leads to relations of the form $\alpha_{i} D_{1}+\beta_{i} D_{2} \sim R_{i}=\sum_{j} e_{i j} P_{j}$. Once we have encountered $w+1$ distinct relations, we apply linear algebra modulo $r$ to obtain a non-trivial linear combination $\sum_{i=1}^{w+1} \gamma_{i}\left(e_{i 1}, e_{i 2}, \ldots, e_{i w}\right)=(0,0, \ldots, 0)$. This yields the discrete $\operatorname{logarithm} \log _{D_{1}} D_{2}=-\left(\sum \gamma_{i} \alpha_{i}\right) /\left(\sum \gamma_{i} \beta_{i}\right) \bmod r$, if $\sum \gamma_{i} \beta_{i}$ is invertible modulo $r$.

The pseudo-random walk is constructed by first computing 20 random reduced divisors $T_{i} \sim a_{i} D_{1}+b_{i} D_{2}$ for $0 \leq i \leq 19$, where $a_{i}, b_{i}$ are randomly selected integers from [0,r-1]. The starting point of the walk is a reduced divisor $R_{0} \sim \alpha_{0} D_{1}+\beta_{0} D_{2}$, where $\alpha_{0}$ and $\beta_{0}$ are randomly selected from $[0, r-1]$. In each step of the pseudorandom walk, the reduced divisor $R_{i}$ is derived from the previous reduced divisor $R_{i-1}=\operatorname{div}(a, b)$ as $R_{i} \sim R_{i-1}+T_{j}$, where $j$ is obtained by taking the integer formed from the five least significant bits of the binary representation of $a$ and reducing it modulo 20. We then obtain a representation $R_{i} \sim \alpha_{i} D_{1}+\beta_{i} D_{2}$ where $\alpha_{i}=\left(\alpha_{i-1}+a_{j}\right) \bmod r$ and $\beta_{i}=\left(\beta_{i-1}+b_{j}\right) \bmod r$. Thus the pair $\left(\alpha_{i}, \beta_{i}\right)$ can be easily computed from the pair $\left(\alpha_{i-1}, \beta_{i-1}\right)$. For the high-genus examples we are interested in, NUCOMP as described in [26] is the most efficient choice for divisor arithmetic.

In order to find relations, each divisor produced by the random walk must be tested for smoothness. Suppose that the factor base contains all prime divisors with degree $\leq t$. Given a reduced divisor $D=\operatorname{div}(a, b), a(u)$ is first subjected to a square-free factorization algorithm (e.g., see [3]). The square-free portion $\bar{a}(u)$ is then tested for $t$-smoothness using the fact that $x^{q^{l}}-x$ is the product of all monic irreducible polynomials in $\mathbb{F}_{q}[x]$ of degree dividing $l$. If $\bar{a}(u)$ is indeed $t$-smooth, then the factorization can be obtained using, for example, the Cantor-Zassenhaus factoring algorithm [6].

Note that it is straightforward to parallelize this algorithm. Each processor independently executes a different random walk and sends the relations to a master process responsible for coordinating the algorithm.

\section{Improvements to THE EnGE-GAUdRY ALGORITHM}

Thériault [39] describes two improvements to Gaudry's index-calculus algorithm [14] for solving the HCDLP on low-genus hyperelliptic curves. In this case, the factor base consists only of degree 1 prime divisors. Thériault shows how the use of 
large primes, as previously employed in integer factorization and discrete logarithm computation in finite fields and class groups of number fields, and a novel idea of using only a portion of the degree 1 prime divisors in the factor base, can be used to obtain algorithms with improved asymptotic run times over 14 .

Let $r$ be a parameter such that $2 / 3<r<1$. Then prime divisors are added to the factor base until $|S|=q^{r}$. In the remainder of this section we will use the term smooth to mean smooth over $S$. We will use $\mathcal{P}$ to denote all prime divisors that have norms with degree equal to one. A potentially smooth divisor is one that is smooth over $\mathcal{P}$ (but not necessarily over $S$ ). A divisor is almost smooth if all of its factors are in $S$ with the exception of one, which is in $\mathcal{P} \backslash S$. That is, a divisor $D$ is almost smooth if $D \sim P+\sum_{i=1}^{|S|} e_{i} P_{i}$, where the divisors $P_{i}$ are again the prime divisors in $D$, and $P \in \mathcal{P} \backslash S$. Such a prime divisor $P$ is called a large prime.

If two almost smooth divisors share the same large prime divisor or one has the large prime $P$ and the other has its inverse $-P$, then we have an intersection, and these two divisors can be combined to form a relation. Let $E_{1} \sim P+\sum_{i=1}^{|S|} e_{1, i} P_{i} \sim$ $a_{1} D_{1}+b_{1} D_{2}$ and $E_{2} \sim P+\sum_{i=1}^{|S|} e_{2, i} P_{i} \sim a_{2} D_{1}+b_{2} D_{2}$. Then

$$
E_{1}-E_{2} \sim \sum_{i=1}^{|S|}\left(e_{1, i}-e_{2, i}\right) P_{i} \sim\left(a_{1}-a_{2}\right) D_{1}+\left(b_{1}-b_{2}\right) D_{2},
$$

and we have a relation. If $E_{2}$ has large prime $-P$, we consider $E_{1}+E_{2}$.

This gives rise to two variations of the random walk strategy as used by Gaudry in [14. The first is almost exactly the same as the original. Each divisor is checked to see if it is potentially smooth. If so, it is completely factored and if the factors are all in $S$ a relation has been found. Otherwise, the divisor is discarded and the next one is tested. The effect of this strategy is that relations will be harder to find as compared with Gaudry's algorithm, but the factor base is smaller, allowing the linear system to be solved faster. By balancing the time for finding relations with the linear algebra, Thériault obtained an improved asymptotic run time.

The second variation that Thériault analyzes takes advantage of the almost smooth divisors. Again, a divisor is first checked for smoothness over $\mathcal{P}$. If it passes this first test, then it is completely factored. If the divisor turns out to be smooth over $S$, a relation is recorded. If the divisor is almost smooth, then the corresponding prime divisor is tested for intersections with any previously found almost smooth divisors. If so, a relation is formed and recorded, with the new almost smooth divisor being removed. Otherwise, the almost smooth divisor is saved and the next divisor is checked.

We now describe our method of incorporating these ideas into the Enge-Gaudry algorithm as described in the previous section. Similarly to that done by Thériault in [39, we introduce a parameter $r$ such that $0<r \leq 1$. Then we create a factor base that contains all prime divisors with degree up to and including $t-1$. In addition, if there are $A_{t}$ prime divisors with degree equal to $t$, we add $r A_{t}$ of them to the factor base. Then the size of the factor base is $\sum_{i=1}^{t-1} A_{i}+r A_{t}$. Note that the previous definitions of smooth and potentially smooth carry over. We let $\mathcal{P}$ denote not the prime divisors with degree equal to 1 , but rather all of the prime divisors with degree up to and including $t$.

The random walk operates in a similar manner as before. A divisor is tested for potential smoothness over $\mathcal{P}$ using the test described in the previous section. If 
the $\operatorname{divisor} \operatorname{div}(a, b)$ passes this first test, then $a$ is explicitly factored to see if it is actually smooth over the norms of the prime divisors in the factor base.

Recall that this is essentially how Thériault's algorithm works. The change in what is included in the factor base obviously results in a change in how the large prime variations work. We consider two different ideas for incorporating large primes into the large genus case.

First we describe the obvious generalization of Thériault's work. Suppose we let $r$ be such that $0<r<1$ as above. Then there will be some prime divisors with degree equal to $t$ that are not in the factor base. Similar to [39], these are our large primes. These large prime divisors work in the exact same manner as described above. A divisor $\operatorname{div}(a, b)$ is tested for smoothness over the factor base using the test from the previous chapter. If it passes, then $a$ is factored, and if the divisor turns out to be almost smooth, we check for intersections. If this results in a relation being formed, then it is added to the collection of relations that have been found. Otherwise the almost smooth divisor is stored. If the tested divisor is neither smooth nor almost smooth, it is discarded.

The second idea is to fix the degree bound for the divisors in the factor base to be $t$ and include in the set $\mathcal{P}$ the prime divisors with norms having degree $t+1$. We allow our $r$ parameter to be in the range $(0,1]$ and thus the large prime divisors include all of the prime divisors with degree equal to $t+1$ and possibly prime divisors with degree $t$, if $r$ is not equal to 1 . We proceed in a similar manner. Test a divisor for smoothness over $\mathcal{P}$. If it passes, factor the divisor. If it is almost smooth, check for intersections and form relations or store the almost smooth divisor.

3.1. Empirical analysis. In [25, empirical estimates are provided for the expected number of random walk steps required to find all required relations. We now extend this analysis to incorporate our adaptations of Thériault's improvements. Given empirical data about the amount of time required to generate and test a divisor, these computations can give estimated runtimes for various combinations of $t, r$ and different strategies for applying the large prime variant. Furthermore, if estimated running times for the linear algebra with different sized matrices are available, we can compute estimated times for solving the discrete logarithm problem. We apply these methods to concrete examples in Section 5 .

We first recap the analysis developed by Jacobson, Menezes, and Stein [25] for the Enge-Gaudry algorithm without $r$ and large primes. Assume that we are working with a hyperelliptic curve of genus $g$ defined over $\mathbb{F}_{q}$. Let $A_{l}$ be the number of irreducible polynomials $p$ of degree $l$ for which there exists a prime $\operatorname{divisor} \operatorname{div}(p, b)$, where $1 \leq l \leq t$. From [42, Chapter 14] we know that there are

$$
I(l, q)=\frac{1}{l} \sum_{d \mid l} \mu\left(\frac{l}{d}\right) q^{d}
$$

monic irreducible polynomials of degree $l$ in $\mathbb{F}_{q}[X]$, where $\mu(n)$ is the Möbius function. Recall that $\mu(n)$ evaluates to 1 if $n=1,(-1)^{k}$ if $n$ is the product of $k$ distinct primes and 0 if $n$ is not square free. We expect that for about half the irreducible polynomials $p$, there exists $b \in \mathbb{F}_{q}[X]$ such that $p \mid b^{2}+h-f$ [42], so that

$$
A_{l} \approx \frac{1}{2}\left(\frac{1}{l} \sum_{d \mid l} \mu\left(\frac{l}{d}\right) q^{d}\right) .
$$


The multiplier $1 / 2$ comes from the fact that we obtain two prime divisors from each irreducible polynomial that splits, and that they are inverses of each other; one of these becomes an element in the factor base.

Note that this estimate is rather rough, as the Hasse-Weil bound implies that $A_{l}=1 / 2(I(l+q)+O(\sqrt{I(l, q)}))$, and the error in our estimate is therefore not negligible. For the HCDLP instances with which we were concerned in this paper, our crude estimate worked reasonably well, However, refining the analysis using an exact value of $A_{l}$ for a particular curve could yield improvements, especially if the size of the Jacobian is near either endpoint of the Hasse-Weil interval.

As in [25], and generalized in [32] by Maurer, Menezes and Teske, let $M(g, t)$ denote the number of $t$-smooth reduced divisors with norms having degree at most $g$. Then we get

$$
M(g, t)=\sum_{i=1}^{g}\left(\left[x^{i}\right] \prod_{l=1}^{t}\left(\frac{1+x^{l}}{1-x^{l}}\right)^{A_{l}}\right),
$$

where we use $\left[x^{i}\right]$ to denote the coefficient of $x^{i}$. When $A_{l}$ is known, $M(g, t)$ can be computed by finding the first $g+1$ terms of the Taylor expansion of $\prod_{l=1}^{t}\left(\frac{1+x^{l}}{1-x^{l}}\right)^{A_{l}}$ about $x=0$ and summing the coefficients of $x, x^{2}, \ldots, x^{g}$.

We assume that reduced $t$-smooth divisors are distributed evenly in the Jacobian. With this assumption we can compute the expected number of random walk iterations needed to find a reduced $t$-smooth divisor. We denote this value by $E(t)=\# J_{C}(k) / M(g, t)$. Since each splitting polynomial gives rise to a prime divisor in the factor base, let $F(t)=\sum_{l=1}^{t} A_{l}$ be the size of the factor base. In 25] the authors find $F(t)+5$ relations before performing the linear algebra step, a decision made based on empirical data. We use the same value here. Then we expect to create and test $T(t)=(F(t)+5) E(t)$ divisors to find a sufficient number of relations.

Adding the parameter $r$. If we introduce the parameter $r$ only and do not use large primes, as in the first variant analyzed by Thériault in [39], the only change is the number of prime divisors with degree $t$ in the factor base. Thus, we repeat the above calculations using $r A_{t}$ in place of $A_{t}$ and obtain $M(g, t), E(t)$, and $F(t)=\sum_{l=1}^{t-1} A_{l}+r A_{t}$ as before.

Adding large primes and setting $r=1$. We now explain how we can estimate the number of random walk steps needed when using the large prime versions of the algorithm. We start with the most basic situation: setting $r=1$ and using the prime divisors of degree $t+1$ as large primes. During the search for relations we encounter both smooth relations and almost smooth relations. We have to consider how many of each are encountered in the search.

The number of $t$-smooth divisors is still $M(g, t)$ as defined above. We also need to count the number of almost smooth divisors. An almost smooth divisor is one that is smooth over the prime divisors of degree less than or equal to $t$, with the exception of a single factor which has degree equal to $t+1$. Then the number of almost smooth divisors is the number of $t$-smooth divisors with degree less than or equal to $(g-(t+1))$, given by $M(g-(t+1), t)$, multiplied by the number of prime divisors with degree equal to $t+1$. A divisor with degree equal to $(g-(t+1))$ added to a divisor with degree equal to $t+1$ has degree less than or equal to $g$, so the 
divisors we are counting are reduced, as are the divisors produced by the random walk that are tested for smoothness.

Recall that $A_{l}$ represents the number of irreducible polynomials of degree $l$ and each irreducible polynomial gives rise to two distinct prime divisors - a divisor and its negation. Then there are $2 A_{t+1}$ large prime divisors and a total of $A(g, t)=2 A_{t+1} M(g-(t+1), t)$ almost smooth divisors. We assume that these are also randomly distributed amongst the set of effective degree zero divisors. Then, the number of steps required to find an almost smooth divisor is $E_{L P}(t)=\# J_{C}(k) / A(g, t)$ and the expected number of steps required to find a smooth divisor is $E(t)=\# J_{C}(k) / M(g, t)$ as described above.

If in the course of our search we find $x$ almost smooth divisors, then we expect that in $x E_{L P}(t)$ random walk steps we would also find $x \frac{E_{L P}(t)}{E(t)}=x \frac{M(g, t)}{A(g, t)}$ smooth divisors. We need to compute the number of relations we expect to obtain by combining the $x$ almost smooth divisors. In [39, Section 5.6], Thériault provides us with the required information. Let $E_{n, s}$ be the expected number of intersections when $s$ samples are drawn with replacement from a set of $n$ elements. Then from [39, Theorem 1] we know that when $3 \leq s<n / 2, \frac{2 s^{2}}{3 n} \leq E_{n, s} \leq \frac{s^{2}}{n}$. Here we have $n=2 A_{t+1}$, the number of possible large prime divisors, and $s=x$ is the number of almost smooth divisors found. The number of intersections, $E_{n, s}=E_{2 A_{t+1}, x}$, is thus the number of relations we expect to find from the almost smooth divisors.

Again, we would like the search to yield a total of $F(t)+5$ relations. Still using $x$ to represent the number of almost smooth divisors found, we need $x$ such that

$$
x \frac{M(g, t)}{A(g, t)}+\frac{2}{3} \frac{x^{2}}{2 A_{t+1}}=F(t)+5 .
$$

Solving for $x$ and taking the positive root gives

$$
x=\frac{\sqrt{9 M(g, t)^{2}\left(2 A_{t+1}\right)^{2}+48 F(t) A(g, t)^{2} A_{t+1}+120 A(g, t)^{2} A_{t+1}}-3 M(g, t) A_{t+1}}{4 A(g, t)} .
$$

This gives us an expected value of $T(t)=x E_{L P}(t)$ random walk steps needed to generate the relations for the linear system.

Using large primes with $r \neq 1$ and degree $t$ polynomials. We now consider the computations in the case where we allow $r$ to vary and use only the prime divisors having norms with degrees equal to $t$ not in the factor base as the large primes. Let $L(t, r)=A_{t}-r A_{t}$ denote the number of irreducible polynomials that generate large prime divisors resulting from fixing $r$. Then as before we have $2 L(t, r)$ large prime divisors and $A(g, t)=2 L(t, r) M(g-t, t)$ almost smooth divisors, where $r A_{t}$ is used in place of $A_{t}$ in computing $M(g-t, t), M(g, t)$ and $F(t) . E_{L P}(t)=N / A(g, t)$ as before. We then find $x$ using the same method as above and represent the number of divisors we expect to be tested by $T(t)=x E_{L P}(t)$.

Using large primes with $r \neq 1$ and degree $t+1$ polynomials. Finally, we consider the computations for the case where we allow $r$ to vary and use both the remaining prime divisors with degree equal to $t$ along with the prime divisors with degree equal to $t+1$ as large primes. Then $L(t, r)=A_{t}-r A_{t}$ is as above and $A(g, t)=$ $2 L(t, r) M(g-t, t)+2 A_{t+1} M(g-(t+1), t)$ is the number of large prime divisors, where again $M(g-t, t), M(g-(t+1), t)$ and $F(t)$ are all computed using $r A_{t}$ in place of $A_{t}$. Once again, compute $E_{L P}(t)=N / A(g, t), x$ and $T(t)=x E_{L P}(t)$ using the previous method. 


\section{A SIEVE-BASED ALGORITHM}

In 41, Vollmer introduced a subexponential algorithm for computing discrete logarithms in the class group of an imaginary quadratic number field. This algorithm was of interest because it did not require the class number a priori. In our setting we assume that the class number $\# J_{C}(k)$ is available, but the interesting aspect of Vollmer's algorithm is that it is amenable to an especially efficient sieve-based relation generation strategy in the number field case. In this section we describe a version of Vollmer's algorithm for the HCDLP. We also describe an improved version of the sieving methods of Flassenberg and Paulus [1] that is suitable to our setting.

Vollmer's algorithm [41, given divisors $D_{1}$ and $D_{2}$ such that $D_{2}=s D_{1}$, computes $s$ as follows:

(1) Generate a factor base $S=\left\{P_{1}, \ldots, P_{k}\right\}$ with $\operatorname{deg} P_{i} \leq t$ as described above.

(2) Randomly generate $k+5$ relations $\vec{e}_{j}=\left(e_{1, j}, \ldots, e_{k, j}\right)$ such that

$$
\sum_{i=1}^{k} e_{i, j} P_{i} \sim \operatorname{div}(1,0) .
$$

Set $A=\left(e_{i, j}\right)$.

(3) Find vectors $\vec{v}_{-D_{1}}=\left(v_{-D_{1}, 1}, \ldots, v_{-D_{1}, k}\right)$ and $\vec{v}_{D_{2}}=\left(v_{D_{2}, 1}, \ldots, v_{D_{2}, k}\right)$, such that $-D_{1} \sim \sum_{i=1}^{k} v_{-D_{1}, i} P_{i}$ and $D_{2} \sim \sum_{i=1}^{k} v_{D_{2}, i} P_{i}$. Note that we have $-D_{1}+\sum_{i=1}^{k}-v_{-D_{1}, i} P_{i} \sim \operatorname{div}(1,0)$ and $D_{2}+\sum_{i=1}^{k}-v_{D_{2}, i} P_{i} \sim \operatorname{div}(1,0)$, so the vectors $\left(1,0,-\vec{v}_{-D_{1}}\right)$ and $\left(0,1,-\vec{v}_{D_{2}}\right)$ are relations over the extended factor base $\left\{-D_{1}, D_{2}, P_{1}, \ldots, P_{k}\right\}$.

(4) Set $\bar{A}=\left(\begin{array}{ccc}1 & 0 & \overrightarrow{0} \\ 0 & 1 & \overrightarrow{0} \\ -\vec{v}_{-D_{1}}^{T} & -\vec{v}_{D_{2}}^{T} & A\end{array}\right)=\left(\begin{array}{c}\vec{a} \\ A^{\prime}\end{array}\right)$ and solve the linear system $A^{\prime} \vec{v}=$ $(1,0,0,0, \ldots, 0)^{T}$ over the integers modulo $N$

(5) Set $s \equiv \vec{v} \cdot \vec{a} \bmod N$.

We show that $s$ is indeed the discrete logarithm. If $A^{\prime} \vec{v} \equiv(1,0,0,0, \ldots, 0)^{T}$ $(\bmod N)$ has a solution, then we can compute $\bar{A} \vec{v} \equiv(s, 1,0, \ldots, 0)^{T}(\bmod N)$, and thus $(s, 1,0, \ldots, 0)$ is a linear combination of the columns of $\bar{A}$. This implies that $-s D_{1}+D_{2} \sim \operatorname{div}(1,0)$, since the columns of $\bar{A}$, when taken as coefficients of a linear combination on the extended factor base $\left\{-D_{1}, D_{2}, P_{1}, \ldots, P_{k}\right\}$, also result in $\operatorname{div}(1,0)$. Finally, since $-s D_{1}+D_{2} \sim \operatorname{div}(1,0)$, we know that $D_{2} \sim s D_{1}$, giving us the correct result.

Since we are computing relations randomly, there are circumstances in which this could fail. Consider the homomorphism $\phi: \mathbb{Z}^{k} \rightarrow J_{C}(k)$ that takes vectors $\vec{v}$ in $\mathbb{Z}^{k}$ to the linear combination over the factor base $\sum_{i=1}^{k} v_{i} P_{i}$. The set of relations, i.e., those vectors $\vec{v}$ for which this linear combination results in $\operatorname{div}(1,0)$, forms the kernel of $\phi$, and we have $\mathbb{Z}^{k} / \operatorname{ker} \phi \cong J_{C}(k)$, provided that the factor base generates the entire Jacobian. If the lattice generated by the columns of $A$ is not equal to the above kernel, then it is possible that we cannot find a solution vector $\vec{x}$.

In an attempt to avoid this situation, we can take some measures to make it more likely that the sublattice generated by the columns of $A$ has full rank. For example, it is straightforward to ensure that multiples of existing relations are discarded. One could also generate relations such that each member of the factor base is used in 
at least one relation. This can still be done randomly, as discussed later, but slows down computations considerably.

In 41, Vollmer analyzes his algorithm in the quadratic number field setting and shows that its run time is subexponential in $\log |\Delta|$, where $\Delta$ is the discriminant of the field. In the hyperelliptic curve setting, assuming we know $\# J_{C}(k)$, we expect an asymptotic result similar to that of Enge and Gaudry, but the work is left for future consideration.

4.1. Sieving. An alternative method of relation generation in hyperelliptic curves is motivated by the successful use of sieving in factoring, such as in 36 and 30. Sieving is used to generate relations efficiently for computing class groups in quadratic number fields by Jacobson in 22 and by Jacobson to solve discrete logarithms in quadratic number fields in 24]. In [11, Flassenberg and Paulus provide an algorithm for sieving in hyperelliptic curves with characteristic not equal to 2 .

In the following we describe how sieving can be employed to produce the relations required for Vollmer's method to solve the HCDLP, by generalizing the approach in [11] to the characteristic 2 case. Given the practical improvements achieved via sieving in other settings, we hope to obtain improvements in our setting as well. In Section 5, we describe empirical results showing that in our examples this is indeed the case.

The basic idea behind using sieving to generate relations, generalized to our context, is as follows. We use the well-known correspondence between divisors of a hyperelliptic curve $C$ and integral ideals of the corresponding function field (see, for example, [7, Sec. 4.4.6]). Recall that we have $v^{2}+h v=f$, where $f$ is monic with $\operatorname{deg}(f)=2 g+1$, and since we are considering the characteristic 2 case, $h \neq 0$ has degree at most $g$. Suppose we have a $\operatorname{divisor} D=\operatorname{div}(a, b)$ that is known to be smooth over the factor base. The divisor $D$ corresponds to the ideal $\mathfrak{a}=a \mathbb{F}_{q}[X]+(b+v) \mathbb{F}_{q}[X]$. Let $\alpha=a S+(b+v) T \in \mathfrak{a}$ with $S, T \in \mathbb{F}_{q}[X]$ (where $\left.a \mid b^{2}+b h+f\right)$ be an arbitrary element in $\mathfrak{a}$. Then $N(\alpha)$, the product of $\alpha$ and its conjugate, is

$$
(a S+(b+v) T)(a S+(b+h+v) T)=a\left(a S^{2}+h S T+\frac{b^{2}+b h+f}{a} T^{2}\right) .
$$

Since $a=N(\mathfrak{a})$ there exists an ideal $\mathfrak{b}$ with $N(\mathfrak{b})=F(S, T)=a S^{2}+h S T+c T^{2}$, where $c=\frac{b^{2}+b h+f}{a}$ such that $\mathfrak{a b}=(\alpha)$. In terms of divisors, this means that there exists a divisor $D^{\prime}$ corresponding to $\mathfrak{b}$ such that $D+D^{\prime}=\operatorname{div}(\alpha)$ (i.e., $D+D^{\prime}$ is principal) and $N\left(D^{\prime}\right)=F(S, T)$. If $F(S, T)$ is smooth for some $S, T \in \mathbb{F}_{q}[x]$, then we can factor $D^{\prime}$ over the factor base. We then have that $D+D^{\prime}=\operatorname{div}(\alpha)$ is a relation, because both $D$ and $D^{\prime}$, and hence $D+D^{\prime}$ factor over the factor base.

Thus, given a smooth divisor $D$, finding relations reduces to finding smooth values of $F(S, T)$. Furthermore, for a single fixed divisor $D$, every pair $S, T$ such that $F(S, T)$ is smooth yields a different relation. Sieving allows us to find such smooth values efficiently. In the context of relation generation for Vollmer's algorithm, we repeat the process with different smooth divisors $D$ until the required number of relations (at least as many as the size of the factor base) have been found.

We consider one-dimensional sieving, as in 23. Fix $T=1$, and let $F(S)=$ $a S^{2}+h S+c$ be the sieving polynomial with coefficients in $\mathbb{F}_{q}[X]$. Fix a prime divisor $P$ from the factor base and consider $N(P)=p$. Let $r$ be a root of $F(S)$ modulo $p$. Then $F(r)$ is divisible by $p$, as is $F(z)$ for $z=r+k p$ for $k \in \mathbb{F}_{q}[X]$. 
To illustrate the basic functionality of sieving, we first describe the case where $F(S) \in \mathbb{Z}[x]$. Our approach is modeled after that described in Section 3.2.5 and Section 6.1 .2 (point 3 on p. 231) of 9 . We begin by selecting a sieve interval $[-M, M]$, where $M$ is a positive integer, and initializing the sieve array $A$ by setting $A[z]=0$ for $z=-M, \ldots, M$. Next, for each prime $p$, compute the roots of $F(S)$ modulo $p$ (there could be one or two roots), and for each root $r$, add $\log p$ to $A[r+k p]$ for $k \in \mathbb{Z}$ such that $-M \leq r+k p \leq M$. After all primes have been processed, traverse through the sieve array and any $z$ such that $A[z]$ is larger than a given tolerance value $Y$ is marked as a candidate. For each candidate $z$, compute $F(z)$ and test it for smoothness over the factor base. Any smooth candidates result in relations.

The ideas of adding $\log p$ to $A[z]$ for which $p \mid F(z)$ and using a tolerance value to detect smooth values of $F$ are intended to increase efficiency at the cost of precision in the smoothness testing. One completely accurate version of sieving would be to initialize each sieve array entry to one, multiply $A[z]$ by $p^{e}$ whenever $p^{e} \| F(z)$, and to deem $F(z)$ smooth whenever the value in $A[z]$ is exactly equal to $F(z)$. The first improvement, adding $\log p$ instead of multiplying by $p$ will be faster than multiplication, but introduces some inaccuracy due to round-off error. Using a tolerance value to detect candidates $z$ for smooth $F(z)$ avoids the problem of accurate approximations at the cost of possibly testing non-smooth values of $F(z)$.

The second improvement is to only add $\log p$ to $A[z]$ when $p \mid F(z)$ and not worry about higher powers. The result is that after sieving, the values stored in $A[z]$ will be less than $\log F(z)$ whenever a higher power of $p$ divides $F(z)$. The tolerance value approach allows us to circumvent this problem. By carefully selecting this value in such a way that it is less than the minimum value expected for a completely smooth $F(z)$, smooth values involving higher powers of factor base primes will also be detected at the cost of introducing false positives $(A[z]$ exceeds the tolerance value but $F(z)$ is not smooth). Through careful optimization, a balance can be found between the amount of time spent testing non-smooth values and the time saved by not explicitly testing for higher powers.

Another benefit of the tolerance value approach is that it simplifies the search for large prime relations. Recall from Section 3 that a large prime relation is completely smooth except for one prime factor outside of the factor base. By setting the tolerance value even lower, we can also identify candidates $z$ for which $F(z)$ is smooth except for one additional prime factor. This simplifies the detection of large prime relations at the expense of introducing false positives, and empirical optimization can be used to find a balance and minimize the overall run time.

These ideas can be generalized to the case of function fields easily, using the fact that the size of $F(z) \in \mathbb{F}_{q}[X]$ is measured by its degree. Thus, the sieve array entries are initialized to zero, and we add $\operatorname{deg}(p)$ to $A[z]$ for every index $z$ such that $p \mid F(z)$. Although adding degrees does not introduce imprecision via round-off errors, we can still make use of the tolerance value approach to account for prime powers and large primes exactly as before.

As Flassenberg and Paulus point out in [11], sieving in the hyperelliptic curve context provides a new challenge: representing and moving through a sieve array where all values involved are polynomials. In the integer case we can represent the sieve array by integer indices of an array, as the map from $[-M, M]$ to these indices is trivial. However, in the hyperelliptic curve context it is not as clear how we can 
map polynomials in $\mathbb{F}_{q}[X]$ to these integer indices efficiently. Additionally, moving from index $r+k p$ to $r+(k+1) p$ in the integer case is easy as we just move $p$ places in the array. Again, in the hyperelliptic curve context we do not have this luxury because the $k$ values are in $\mathbb{F}_{q}[X]$, and the distance between indices corresponding to $r+k p$ and $r+k^{\prime} p$, where $k^{\prime}$ follows $k$ in whatever ordering we choose amongst these polynomials, is in general not constant.

We now present an improved version of Flassenberg and Paulus' sieving strategy suitable to the characteristic 2 case. As before, we continue to consider onedimensional sieving, and set $F(S)=F(S, 1)$ to be the sieving polynomial. In this case we can set $b_{F}=a S+b$ and find the signs of the exponents in $\vec{v}$ by finding $s_{i}$ such that $s_{i}=1$ if $b_{F} \equiv b_{p_{i}}\left(\bmod p_{i}\right)$ and $s_{i}=-1$ if $b_{F} \equiv b_{p_{i}}+h\left(\bmod p_{i}\right)$ for all prime divisors $P_{i}$ in the factor base with $N\left(P_{i}\right)=p_{i}$.

Since we are working over a characteristic 2 field, we cannot use the familiar quadratic formula to find the roots of $F(S) \bmod p$, but we can instead take advantage of the linearity and bijectivity of the squaring operation. If $p \mid a$, then

$$
F(S) \equiv h S+c \quad(\bmod p),
$$

and $r \equiv c h^{-1}(\bmod p)$ is clearly the single root. If $p \mid f$ and $p \nmid a$, then

$$
F(S) \equiv a S^{2}+h S+b^{2}\left(a^{-1}\right)+b h\left(a^{-1}\right) \quad(\bmod p),
$$

and the roots are given by $r \equiv b a^{-1}(\bmod p)$ and $r \equiv(b+h) a^{-1}(\bmod p)$, by inspection. Finally, if $p \nmid a$ and $p \nmid f$, then we can solve

$$
r^{2}+r h a^{-1}+c a^{-1} \equiv 0 \quad(\bmod p)
$$

using a generalized version of the (RESSOL) algorithm by Shanks as in [11. This gives us the roots $X$ and $X+h a^{-1}(\bmod p)$. Thus we have the roots of the sieving polynomial and can proceed with the rest of the algorithm.

Sieve array implementation. Efficiently moving through the sieve array given a root is one of the main complications in the hyperelliptic curve case. As originally presented in [11, we assign a sieve array for which each element of the array corresponds to a polynomial $z \in \mathbb{F}_{q}[X]$ with $\operatorname{deg}(z) \leq M$ for some degree bound $M$. Each such polynomial $z$ is mapped to a unique index $\nu(z) \in\left[0, \ldots, q^{M}-1\right]$ as follows. We define a map $\nu_{0}: \mathbb{F}_{q} \rightarrow \mathbb{Z}^{\geq 0}$ that takes every element $\gamma \in \mathbb{F}_{q}$ to a unique integer between 0 and $q-1$. If the elements of $\mathbb{F}_{q}$ are represented as polynomials, this is done by evaluating the polynomial at the characteristic of the field. Then the map $\nu: \mathbb{F}_{q}[X] \rightarrow \mathbb{Z}^{\geq 0}$ is defined for $z=z_{0}+z_{1} X+\cdots+z_{d} X^{d} \in \mathbb{F}_{q}[X]$, where $d=\operatorname{deg}(z)$, by $\nu(z)=\sum_{i=0}^{d} \nu_{0}\left(z_{i}\right) q^{i}$.

Let $P$ be a prime divisor in the factor base of norm $p$, and $r$ a root of $F(S) \bmod p$. Moving through the sieving array from one index to the next requires the computation of $\nu\left(r+k_{j+1} p\right)$ given $\nu\left(r+k_{j} p\right)$ where $k_{0}, k_{1}, \ldots$ represents an ordering of the polynomials $k$ such that $\operatorname{deg}(r+k p) \leq M$. Our method, based on that of [1], is as follows. We first compute $z_{j+1}=r+k_{j+1} p$ from $z_{j}=r+k_{j} p$, observing that $z_{j+1}=z_{j}+\delta$, where

$$
\delta=z_{j+1}-z_{j}=\left(k_{j+1}-k_{j}\right) p .
$$

Unfortunately, $\nu$ is not additive, and in particular $\nu\left(z_{j+1}\right)=\nu\left(z_{j}+\delta\right) \neq \nu\left(z_{j}\right)+\nu(\delta)$. Thus, we evaluate $\nu\left(z_{j+1}\right)$ by computing $\delta=\left(k_{j+1}-k_{j}\right) p$, computing $z_{j+1}=z_{j}+\delta$, and finally by evaluating $\nu\left(z_{j+1}\right)$ directly from $z_{j+1}$. This latter process can be sped up by using a precomputed table of the powers of $q^{i}$ for $0 \leq i \leq M$, and, if $q$ is 
sufficiently small, using table look-up to compute the $\nu_{0}$ values of the coefficients of $z_{j+1}$.

The main difference between our method and that of [11] is that we use a more direct approach to compute $z_{j+1}$ from $z_{j}$. In the case that $q$ is a prime, the coefficients of $z_{j+1}$ are represented simply as integers modulo $q$. As a result, evaluating $\nu_{0}$ is trivial, and the computation of $z_{j+1}$ can be done by carefully updating the coefficients of $z_{j}$. This approach fails in our case as the coefficients of the $z_{j}$ are represented as polynomials in $\mathbb{F}_{p}$, where $q=p^{n}$ and updating coefficients by adding 1 does not work.

Low-degree sieving. The factor base typically consists of a much larger number of prime divisors having norms with large degree than divisors with small degree norms. Considering the norms of these prime divisors, we expect fewer of these higher degree irreducible polynomials to divide the test polynomials. However, a significant amount of time is spent checking for divisibility by these high-degree irreducible polynomials.

This observation leads to the idea of sieving with only the norms with lower degrees. For example, sieve with the norms of prime divisors that have degree up to $t-1$ if the factor base degree bound is $t$. The tolerance value can then be adjusted accordingly to account for the possibility of a higher degree factor that has not been sieved. How we do this is discussed at the end of the section. Clearly, the amount of time spent sieving is reduced, but there is a trade off in that potentially smooth candidates have to be tested for smoothness over the factor base. The hope is that a tolerance value can be chosen such that the extra amount of time spent testing for smoothness is less than the time that would have been spent sieving with the higher degree norms.

Self-initialization. The sieving process relies greatly on the speed at which sieving polynomials can be created and the time taken to compute the roots of these polynomials modulo primes. For factoring [1, 8, and computing class groups in quadratic number fields [23], a process called self-initialization has been introduced which allows the amount of computation done to be reduced significantly. As there are no known implementations of self-initialization being used in conjunction with sieving in the hyperelliptic curve case, we now describe self-initialization as described in 23], generalized to work in this context in characteristic 2.

Suppose we have a divisor $D$ with norm $a$ that is the sum of $j$ distinct prime divisors $Q_{i}$, with norms $q_{i}$. Then $D=\sum_{i=1}^{j} v_{i} Q_{i}$ where $v_{i} \in\{-1,1\}$ and $a=$ $\prod_{i=1}^{j} q_{i}{ }^{\left|v_{i}\right|}$. Note that $\sum_{i=1}^{j}-v_{i} Q_{i}=-D$, so to avoid duplicate relations we fix $v_{j}=1$ and only produce $2^{j-1}$ divisors, denoted by $D_{1}=\operatorname{div}\left(a, b_{1}\right), D_{2}=\operatorname{div}\left(a, b_{2}\right)$, $\ldots, D_{2}^{j-1}=\operatorname{div}\left(a, b_{2^{j}-1}\right)$. The trick is computing these divisors without actually computing the various products of prime ideals. In fact, both this and the computation of most of the roots of the corresponding sieving polynomials modulo the norms of the factor base elements can be computed in an efficient manner.

Let $F_{i}(S)=a S^{2}+h S+c_{i}$ denote the sieving polynomial corresponding to $D_{i}$, where $c_{i}=\left(b_{i}^{2}+b_{i} h+f\right) / a$ and, as noted above, $a=\prod_{i=1}^{j} q_{i}$. Similar to 23, Theorem 4.1], for $1 \leq i \leq j$ we write

$$
B_{i}=\left(a / q_{i}\right)\left(\left(a / q_{i}\right)^{-1} t_{i} \quad\left(\bmod q_{i}\right)\right) \quad(\bmod a)
$$


and

$$
B_{i}^{\prime}=\left(a / q_{i}\right)\left(\left(a / q_{i}\right)^{-1}\left(t_{i}+h\right) \quad\left(\bmod q_{i}\right)\right) \quad(\bmod a)
$$

where $t_{i}$ is a solution to $x^{2}+x h \equiv f\left(\bmod q_{i}\right)$. Then $b= \pm B_{1} \pm B_{2}+\cdots+B_{j}$ is a solution to $x^{2}+x h \equiv f(\bmod a)$, where we use $-B_{i}$ to denote $B_{i}^{\prime}$. To see this, first recall that the Chinese Remainder Theorem tells us that if $a=\prod_{i=1}^{j} q_{i}$, then $b^{2}+b h+f \equiv 0(\bmod a) \Longleftrightarrow b^{2}+b h+f \equiv 0\left(\bmod q_{i}\right)$ for all $1 \leq i \leq j$. Note that $B_{i} \equiv t_{i}\left(\bmod q_{i}\right)$ and $B_{i} \equiv 0\left(\bmod q_{k}\right)$ for $1 \leq k \leq j, k \neq i$. Similar results hold for $B_{i}^{\prime}$. Then $b^{2}+b h+f \equiv t_{i}^{2}+t_{i} h+f \equiv 0\left(\bmod q_{i}\right)$ or $b^{2}+b h+f \equiv$ $\left(t_{i}+h\right)^{2}+\left(t_{i}+h\right) h+f \equiv 0\left(\bmod q_{i}\right)$ for all $1 \leq i \leq j$. Thus $b$ as defined above is a solution to $b^{2}+b h \equiv f(\bmod a)$.

Now we can easily change sieving polynomials. Let $\vec{v}=\left\{v_{1}, v_{2}, \ldots, v_{j}\right\}$ with $v_{i}=1$ for $i=1, \ldots, j$. Then we can compute the sieving polynomial by finding $a=\prod_{i=1}^{j} q_{i}$ and $b_{1}=\sum_{i=1}^{j} B_{i}$, giving $F_{1}(S)=a S^{2}+h S+\left(b_{1}{ }^{2}+b_{1} h+f\right) / a$. Note that we fix $v_{j}=1$ in order to ensure we do not use both $\vec{v}$ and $-\vec{v}$. Now, consider a $(j-1)$-bit Gray code, starting with $\vec{v}$. Then iterating through the possible values for $\vec{v}$ in this manner results in each $\vec{v}_{l}$ differing from $\vec{v}_{l-1}$ in a single index, say $k$. Suppose $D_{l-1}=\left(a, b_{l-1}\right)$ with $b_{l-1}=v_{1} B_{1}+v_{2} B_{2}+\cdots+v_{k} B_{k}+v_{k+1} B_{k+1}+\cdots+B_{j}$. Then $b_{l}=v_{1} B_{1}+v_{2} B_{2}+\cdots+\left(-v_{k}\right) B_{k}+v_{k+1} B_{k+1}+\cdots+B_{j}$ where $-B_{k}=B_{k}^{\prime}$, and we have $b_{l}=b_{l-1}+B_{k}+B_{k}^{\prime}$. This gives $D_{l}=\left(a, b_{l}\right)$ and the sieving polynomial $F_{l}(S)=a S^{2}+h S+\left(b_{l}^{2}+b_{l} h+f\right) / a$ with two additions, assuming the previously computed $B_{i}$ and $B_{i}^{\prime}$ values are stored.

In addition to computing the next sieving polynomial, we can easily compute the roots of the new sieving polynomial modulo the norms of the prime divisors $P$ in the factor base. Let $P$ be an divisor in the factor base such that $P \notin\left\{Q_{1}, \ldots, Q_{j}\right\}$. First, suppose that $N(P)=p, p \nmid a$, and let $r$ be a root of $F_{l}(S)(\bmod p)$. Then $r+\left(B_{k}+B_{k}^{\prime}\right) a^{-1}(\bmod p)$ is a root of $F_{l+1}(S)(\bmod p)$, where $k$ is the index where $\overrightarrow{v_{l}}$ and $\overrightarrow{v_{l+1}}$ differ. This is easy to confirm with substitution, and easy to compute if $\left(B_{k}+B_{k}^{\prime}\right) a^{-1}$ is precomputed. To find the roots for the remaining norms of the prime divisors, that is for $p \mid a$, we have to compute $r \equiv c_{l+1} h-1\left(\bmod p_{i}\right)$ directly. However, there are only $j$ of these roots that need computing. Thus, we can compute new sieving polynomials and their roots efficiently from previously computed polynomials and roots.

4.2. Parameter selection. Sieving introduces a number of parameters into the algorithms for solving discrete logarithm problems. In addition to the factor base bound $t$, we have to consider how large the sieve radius $M$ should be. For this discussion, let $M$ be the degree of the maximum polynomial in the sieve array. We also need to determine the degree of polynomials with which to sieve, the tolerance value $Y$ that decides whether or not we should check a candidate for smoothness, and $j$, the number of prime divisors used to create the divisor that gives rise to a sieve polynomial. Note that all these parameters are positive integers. Our approach for finding values for these parameters was a combination of analysis and empirical work. Analytical work was used to determine ranges in which we should search for parameters. We then ran test programs in which several hundred relations were found using different combinations of parameters. The set of parameters that resulted in the fastest computation was our final choice. In this section we provide 
guidance for choosing these values, assuming that self-initialized sieving is being used.

Selecting the sieving polynomial in such a way that $\operatorname{deg} F(S)$ is as small as possible for all $S$ in the sieving interval is important, as smaller-degree polynomials are more likely to be smooth than those with large degree. With this in mind, we want $a$, the leading coefficient of $F(S)=a S^{2}+h S+c$, to have degree approximately $g-M$, the genus minus the sieve radius. Because $\operatorname{deg}(S) \leq M$, this ensures that $\operatorname{deg} F(S) \leq g+M+1$, since

$$
\operatorname{deg}(F(S)) \leq \max \left(\operatorname{deg}\left(a S^{2}\right), \operatorname{deg}(h S), \operatorname{deg}(c)\right),
$$

and we have

$$
\begin{aligned}
\operatorname{deg}\left(a S^{2}\right) & =\operatorname{deg}(a)+2 \operatorname{deg}(S) \leq(g-M)+2 M=g+M, \\
\operatorname{deg}(h S) & =\operatorname{deg}(h)+\operatorname{deg}(S) \leq g+M,
\end{aligned}
$$

and

$$
\begin{aligned}
\operatorname{deg}(c) & \leq \operatorname{deg}\left(b^{2}+b h+f\right)-\operatorname{deg}(a) \\
& =\max \left(\operatorname{deg}\left(b^{2}\right), \operatorname{deg}(b h), \operatorname{deg}(f)\right)-\operatorname{deg}(a) \\
& \leq \max (2(g-M-1),(g-M-1)+g, 2 g+1)-(g-M) \\
& =\max (g-M-2, g-1, g+1+M) \\
& =g+M+1,
\end{aligned}
$$

since $\operatorname{deg}(b)<\operatorname{deg}(a)=g-M$. In fact, $\operatorname{deg}\left(b^{2}+b h\right)<\operatorname{deg}(f)$, so no cancellation in the numerator of $c$ is possible, and we have $\operatorname{deg}(c)=g+M+1$ for our sieve polynomials.

Since $j$ irreducible polynomials are used to generate $a$, each should have degree $(g-M) / j$. In order to ensure that this is possible, this value must be smaller than the maximum degree of the factor base, $t$, so we must take $j \geq \frac{g-M}{t}$. As a starting point we assume that $t$ is the optimal value determined by the expected random walk values computed using the formulas discussed in Section 3.1. Once we have chosen $M$ and $j$, we can use these to choose which prime divisors are used to generate the sieving polynomials. We simply add any $j$ prime divisors from the factor base with degree equal to $(g-M) / j$ or as close to it as possible.

Using the search method described above, we found empirical data that suggests setting $M=t-2$ and taking $j$ such that $(g-M) / j \approx t-1$ are reasonable settings to use. This makes sense, as we wish to ensure there are enough prime divisors available to generate enough sieving polynomials to find the relations needed. The relationship between $j, M$ and $t$ was used to dictate the parameter search space.

The last parameter is the tolerance value used for sieving. Recall that we only sieve with prime divisors of degree $\leq t-1$ and do not explicitly test for repeated factors, so we need to select a tolerance value that accounts for this. In order to ensure that most of the candidates produced really are smooth, we need to account for not only the degrees of the prime divisors in the factor base but also the degree of $F(S)$ when evaluated at polynomials in the sieve radius and the bound on potential large prime divisors occurring in almost smooth divisors.

We first derive a lower bound on $\operatorname{deg}(F(S))$. For all $S$ with $\operatorname{deg}(S) \leq M$, we have $\operatorname{deg}\left(a S^{2}+h S\right)<\operatorname{deg}(c)$, so $\operatorname{deg}(F(S))=\operatorname{deg}(c)=g+M+1$. 
Now consider some $F(S)$ that is smooth over the factor base. Then $F(S)=$ $\prod_{i=1}^{k} p_{i}^{a_{i}}$ where $p_{i}=N\left(P_{i}\right)$ for $P_{i}$ in the factor base, and $\operatorname{deg} F(S)=\sum_{i=1}^{k} a_{i} \operatorname{deg} p_{i}$. If $a_{i}=1$ for all $i$ and we sieve with all primes in the factor base, then using $g+M+1$ as a tolerance value will cause $F(S)$ to be marked as a candidate, as $\operatorname{deg} F(s) \geq g+M+1$ for all $S$ in the sieve radius. However, if we want to be able to catch factors that are squares, we have to make the tolerance value smaller, for example, $g+1$ would allow for a square factor.

When using large primes, the tolerance value must be smaller to allow for the large prime factor. For example, if we are in the case where the large prime divisors are those with norms of degree $t+1$, we must reduce the tolerance value by $t+1$ because an almost smooth candidate has degree that is the sum of the degrees of the prime divisors in the factor base plus the degree of the large prime, $t+1$ (or $t$, depending on the variation and value of $r$ ). Furthermore, when sieving with only divisors of degree $\leq t-1$, we have to use an even smaller tolerance value, such as $\min (\operatorname{deg}(F(S))-i t=g+M+1-i t$, for some integer $i$, to allow for multiple factors of degree $t$ dividing $F(S)$. This is to account for any missing degree $t$ factors not marked during the sieving process.

These guidelines should be used as a starting point for finding the tolerance value, which can be fine tuned using the tests mentioned above. We used test values in the range $Y \pm i$ where $i$ depended on how long the tests were expected to take, and the results of previous tests, starting with $Y=g-M+1-2 t$.

Certainly, it is not immediately clear if sieving outperforms the random walk method. In the next section, we provide numerical evidence that it can do so when using empirically chosen parameters as described above. A detailed analysis is required to justify, and possibly further refine, our parameter selection strategy and to fully explore sieving.

\section{Numerical RESUlts}

We have implemented our improvements to the Enge-Gaudry algorithm and our sieve-based hyperelliptic curve version of Vollmer's algorithm in C++. NTL 37. is used to perform the finite field arithmetic. An implementation of the Lanczos algorithm from the LinBox package [31] was used to solve the resulting linear systems.

We used the Advanced Cryptography Laboratory (ACL) at the University of Calgary as our testing platform. The ACL is a Beowulf cluster consisting of 152 nodes, 139 of which have dual Intel P4 Xeon $2.4 \mathrm{Ghz}$ processors with $512 \mathrm{~kb}$ cache. The remaining 13 nodes have dual Intel P4 Xeon 2.8 Ghz processors with $512 \mathrm{~kb}$ cache. All nodes have $2 \mathrm{~GB}$ of RAM and $40 \mathrm{~GB}$ hard drives. These nodes are interconnected with gigabit Ethernet. The nodes all run Red Hat Enterprise Linux 3 and have the GNU Multi-Precision C library (GMP) version 4.2.2 ([19]) installed, along with NTL version 5.4.1 ([37]) and the MPICH Message Passing Interface (MPI) version 1.2.5 ([33]). Additionally, we have installed the Automatically Tuned Linear Algebra Software (ATLAS) version 3.7.31 ([2]) and LinBox version 1.1.3 ([31]) to perform linear algebra. The compiler used was GCC version 3.4.4 ([18]).

We now describe some features of our implementations. The relation generation phase is done in parallel in all cases. For the random walk algorithms, each process initializes and performs its own random walk, and all smooth and almost 
smooth relations are reported back to a single "master" process. For the sievebased algorithm, the master process coordinates which sieve polynomials are used by each process by sending a different set of $j$ prime divisors to be used with self-initialization.

Rather than searching the almost smooth divisors for potential intersections each time we find a new one, we wait until the expected number of intersections plus the number of relations currently found is large enough. We use [39, Theorem 1] to determine when this happens. To be exact, we wait until $\frac{x^{2}}{6 A_{t+1}}$ plus the current number of relations found is greater than $F(t)+5$, the total number of relations we wish to find. In this, $x$ is the number of almost smooth divisors found and $A_{t+1}$ is the number of degree $t+1$ irreducible polynomials, which give rise to $2 A_{t+1}$ large prime divisors, divisors, and their inverses, as discussed earlier. Combining the almost smooth relations is done by the master process at this point.

The special relations corresponding to $-D_{1}$ and $D_{2}$ required for Vollmer's algorithm are produced by sieving with divisors of the form $-D_{1}+D^{\prime}$ or $D_{2}+D^{\prime}$ for randomly produced smooth divisors $D^{\prime}$ without using self-initialization. Any smooth value of the corresponding sieving polynomials lead to a factorization of $-D_{1}$ or $D_{2}$. This step is also done in parallel by sending different random divisors $D^{\prime}$ to each process.

Once we have enough relations, we move on to the linear algebra phase. In the Enge-Gaudry algorithm we find a non-zero vector in the kernel of $A$, i.e., we find $\vec{x}$ such that $A \vec{x} \equiv \overrightarrow{0}(\bmod N)$, where $N$ is the provided class number. Note that our implementation assumes $N$ is prime. If not, one can factor $N$ and compute solutions to $A \vec{x} \equiv \overrightarrow{0}$ modulo each factor, combining the results with the Chinese Remainder Theorem, as discussed in [10, Section 4]. We find a solution to this linear system by finding a random vector $\vec{v}$ and using the Lanczos system solver provided by the LinBox library to solve $A \vec{x} \equiv A \vec{v}(\bmod N)$. The vector $\vec{x}-\vec{v}$ is, with high probability, a non-zero vector in the kernel of $A$. We use the function LinBox: : solve using the Lanczos method with the default preconditioner FULL_DIAGONAL, specifying that the matrix is singular, limiting the maximum number of tries to 1 , and ignoring the ability to certify a system without a solution. This last decision was made so that rather than spending time confirming that the system is not solvable, we generate five more relations and try again. Our experiments show that very few additional iterations are required, if any, before a solution is found.

We use the same LinBox function and options to solve the linear system $A^{\prime} \vec{x}=$ $(1,0, \ldots, 0)$ required for Vollmer's algorithm. In addition to computing five new relations, in the event that we cannot solve this linear system, we also recompute the special relations corresponding to $\mathfrak{a}^{-1}$ and $\mathfrak{b}$. Our experiments suggest again that very few, if any, iterations of this process are necessary.

Empirical estimates and parameter selection. We tested our algorithms on instances of the HCDLP in the Jacobian of four genus 31 hyperelliptic curves defined over $k=\mathbb{F}_{q}$ for $q=4,8,16$, and 32. The hyperelliptic curves over these fields, taken from [25], are denoted C62, C93, C124, and C155. They all have $\# J_{C}(k)=2 r$, where $r$ is prime and as described in [25, were obtained by applying the GHS attack to an instance of the ECDLP on elliptic curves defined over $\mathbb{F}_{2^{62}}, \mathbb{F}_{2^{93}}, \mathbb{F}_{2^{124}}$, and $\mathbb{F}_{2^{155}}$, respectively. The curve parameters are listed in Table 1 . The curve equations are given by $v^{2}+h(u) v=f(u)$, where $h, f \in \mathbb{F}_{q}[u]$, and the prime factorizations of $\# J_{C}(k)$ are also listed. 
TABle 1. Hyperelliptic curves C62, C93, C124, and C155 of genus $g=31$.

\begin{tabular}{|l|}
\hline $\mathrm{C} 62, q=4, \mathbb{F}_{2^{2}}=\mathbb{F}_{2}[w] /\left(w^{2}+w+1\right)$ \\
$f(u)=u^{63}+w^{2} u^{62}+u^{48}+w^{2}$ \\
$h(u)=u^{31}+u^{30}+w u^{28}+u^{24}+w^{2} u^{16}+w^{2}$ \\
$\# J_{\mathrm{C} 62}\left(\mathbb{F}_{2^{2}}\right)=2 \cdot 2305843007560748609$ \\
\hline \hline $\mathrm{C} 93, q=8, \mathbb{F}_{2^{3}}=\mathbb{F}_{2}[w] /\left(w^{3}+w+1\right)$ \\
$f(u)=w^{4} u^{63}+w^{5} u^{62}+w^{5} u^{60}+w^{3} u^{56}+w^{5} u^{48}+w u^{32}+w^{5}$ \\
$h(u)=w^{2} u^{31}+w^{5} u^{30}+u^{28}+w^{6} u^{24}+w^{6}$ \\
$\# J_{\mathrm{C} 93}\left(\mathbb{F}_{2^{3}}\right)=2 \cdot 4951760157141611728579495009$ \\
\hline $\mathrm{C} 124, q=16, \mathbb{F}_{2^{4}}=\mathbb{F}_{2}[w] /\left(w^{4}+w+1\right)$ \\
$f(u)=w^{3} u^{63}+w^{7} u^{60}+w^{3} u^{56}+w^{3} u^{48}+1$ \\
$h(u)=w^{9} u^{31}+w^{12} u^{30}+w^{8} u^{28}+w^{13} u^{24}+w^{6} u^{16}+w^{6}$ \\
$\# J_{\mathrm{C} 124}\left(\mathbb{F}_{2^{4}}\right)=2 \cdot 10633823966279326985483775888689817121$ \\
\hline \hline $\mathrm{C} 155, q=32, \mathbb{F}_{2^{5}}=\mathbb{F}_{2}[w] /\left(w^{5}+w^{2}+1\right)$ \\
$f(u)=w^{4} u^{63}+w^{6} u^{62}+w^{15} u^{60}+w^{26} u^{56}+w^{25} u^{48}+w^{7} u^{32}+w^{13}$ \\
$h(u)=w^{2} u^{31}+w^{7} u^{30}+w^{30} u^{28}+w^{22} u^{24}+w^{3} u^{16}+w^{22}$ \\
$\# J_{\mathrm{C} 155}\left(\mathbb{F}_{2^{5}}\right)=2 \cdot 22835963083295358096932727763065266972881541089$ \\
\hline
\end{tabular}

In Section 3.1 we generalized the method of [25] for estimating the number of random divisors that must be tested in order to find a total of $F(t)+5$ relations to methods that also make use of a reduced factor base and large primes. We now apply these ideas to the curves C62, C93, C124, and C155 in order to find optimal parameters for our improved versions of the Enge-Gaudry algorithm. We also estimate the amount of time required to perform the entire algorithm by measuring the time required to create a smaller number of divisors, for example 1000 divisors, and test them for smoothness. With this information we can estimate how long the relation generation stage will take, through interpolating and extrapolating the data. We also have estimated linear algebra times based on some trial runs and extrapolation. Finally, the time required for initialization (that is, creating the factor base) has been measured and used in these estimates. With these timings we can compute estimated runtimes for the Enge-Gaudry algorithm with the curves above and different choices for the factor base bound $t$, the parameter $r$ and what form of almost smooth divisors are considered.

When not using large primes, there are two parameters we can vary: the factor base bound $t$ and the parameter $r$ controlling the number of the largest degree polynomials we use to create prime divisors for our factor base. When using large primes, we consider the case in which we use just the prime divisors having norm with degree equal to $t$ as large prime divisors and the case in which we also include the prime divisors having norms with degree equal to $t+1$.

Tables 2 to 5 provide sample points for each variation of the random walk parameters and each curve we are considering. In these tables $L P$ indicates the number of large prime divisors that exist with these settings, $E(t)$ is the expected number of steps required to find a smooth divisor, $E_{L P}(t)$ is the expected number of tests to find an almost smooth divisor, $T(t)$ is the total number of steps expected to find 
TABLE 2. Estimated C62 runtimes in seconds.

\begin{tabular}{|r|r|r|r|r|r|r|r|r|r|}
\hline$t$ & $r$ & $F(t)$ & $L P$ & $E(t)$ & $E_{L P}(t)$ & $T(t)$ & $T$ & $L A$ & total \\
\hline \multicolumn{1}{|c|}{ Not considering almost smooth divisors } \\
\hline 5 & 1.00 & 144 & - & 36296 & - & 5408075 & 51.61 & 0.07 & 54.14 \\
6 & 0.50 & 309 & - & 7776 & - & 2441636 & 24.13 & 0.34 & 27.24 \\
6 & 1.00 & 474 & - & 2614 & - & 1251873 & 13.20 & 0.81 & 16.97 \\
7 & 0.50 & 1059 & - & 921 & - & 980453 & 10.37 & 4.04 & 19.06 \\
$(*) 7$ & 1.00 & 1644 & - & 421 & - & 694997 & 7.57 & 9.73 & 23.48 \\
\hline Using only degree $t$ norms \\
\hline 5 & 0.90 & 134 & 10 & 48446 & 162924 & 5050630 & 48.67 & 0.07 & 51.21 \\
6 & 0.60 & 342 & 132 & 6092 & 6443 & 1391785 & 13.67 & 0.48 & 17.02 \\
6 & 0.80 & 408 & 66 & 3900 & 9256 & 1249536 & 12.37 & 0.68 & 16.03 \\
6 & 0.90 & 441 & 33 & 3177 & 15884 & 1223078 & 12.15 & 0.79 & 15.97 \\
7 & 0.10 & 591 & 1053 & 2058 & 996 & 802851 & 8.44 & 1.43 & 13.28 \\
\hline Using degree $t+1$ norms
\end{tabular}

TABLE 3. Estimated C93 runtimes in minutes.

\begin{tabular}{|r|r|r|r|r|r|r|r|r|r|}
\hline$t$ & $r$ & $F(t)$ & $L P$ & $E(t)$ & $E_{L P}(t)$ & $T(t)$ & $T$ & $L A$ & total \\
\hline Not considering almost smooth divisors \\
\hline 4 & 1.00 & 596 & - & 1830509 & - & $1.10 \times 10^{9}$ & 178.34 & 0.03 & 178.46 \\
5 & 0.50 & 2234 & - & 145591 & - & 325977380 & 56.30 & 0.45 & 56.88 \\
$(*) 5$ & 1.00 & 3872 & - & 28668 & - & 111146195 & 19.26 & 1.35 & 20.77 \\
6 & 0.50 & 14771 & - & 6281 & - & 92810648 & 17.12 & 19.64 & 37.42 \\
\hline
\end{tabular}

$F(t)+5$ relations, $T$ represents the estimated time required to test $T(t)$ divisors using 256 processors. The column $L A$ refers to the estimated time required for the linear algebra stage. The total time is the sum of $L A, T$, and the time required for initialization (not shown). Those lines marked with $(*)$ represent the settings recommended in 25 .

Note that, when incorporating large primes and $r \neq 1$, the values of $t$ suggested in 25] may not necessarily be the best ones (see C62 and C124). In addition, the use of large primes with appropriate parameter selection results in a significant reduction in the expected number of random walk steps required to generate $F(t)+5$ relations.

Using Tables 2 to 5 we can pick optimal parameters for the Enge-Gaudry algorithm with random walks for relation generation. It would appear that in our 
TABLE 4. Estimated C124 runtimes in hours.

\begin{tabular}{|c|c|c|c|c|c|c|c|c|c|}
\hline$t$ & $r$ & $F(t)$ & $L P$ & $E(t)$ & $E_{L P}(t)$ & $T(t)$ & $T$ & $L A$ & total \\
\hline \multicolumn{10}{|c|}{ Not considering almost smooth divisors } \\
\hline 4 & 0.50 & 4808 & - & 18991245 & - & $9.14 \times 10^{10}$ & 267.29 & 0.07 & 267.37 \\
\hline 4 & 1.00 & 8872 & - & 1498799 & - & $1.33 \times 10^{10}$ & 38.86 & 0.24 & 39.11 \\
\hline 5 & 0.32 & 42426 & - & 267103 & - & $1.13 \times 10^{10}$ & 36.39 & 5.59 & 42.01 \\
\hline 5 & 0.50 & 61300 & - & 127546 & - & $7.82 \times 10^{9}$ & 25.37 & 11.67 & 37.08 \\
\hline (*) 5 & 1.00 & 113728 & - & 25876 & - & $2.94 \times 10^{9}$ & 10.34 & 40.15 & 50.59 \\
\hline \multicolumn{10}{|c|}{ Using only degree $t$ norms } \\
\hline 4 & 0.50 & 4808 & 4064 & 18991245 & 6040905 & $3.60 \times 10^{10}$ & 104.42 & 0.08 & 104.50 \\
\hline 4 & 0.90 & 8059 & 813 & 2308631 & 5163911 & $1.28 \times 10^{10}$ & 37.39 & 0.23 & 37.62 \\
\hline 5 & 0.32 & 42426 & 71302 & 267103 & 88111 & $5.84 \times 10^{9}$ & 18.82 & 6.34 & 25.18 \\
\hline 5 & 0.50 & 61300 & 52428 & 127546 & 67239 & $4.38 \times 10^{9}$ & 14.28 & 13.24 & 27.56 \\
\hline \multicolumn{10}{|c|}{ Using degree $t+1$ norms } \\
\hline 4 & 0.50 & 4808 & 108920 & 18991245 & 1336140 & $3.98 \times 10^{10}$ & 124.06 & 0.08 & 124.15 \\
\hline 4 & 1.00 & 8872 & 104856 & 1498799 & 225558 & $7.72 \times 10^{9}$ & 23.96 & 0.28 & 24.24 \\
\hline 5 & 0.32 & 42426 & 1469042 & 267103 & 27402 & $7.18 \times 10^{9}$ & 24.66 & 6.34 & 31.03 \\
\hline 5 & 0.50 & 61300 & 1450168 & 127546 & 17154 & $5.16 \times 10^{9}$ & 17.80 & 13.24 & 31.09 \\
\hline 5 & 1.00 & 113728 & 1397740 & 25876 & 6964 & $2.28 \times 10^{9}$ & 9.37 & 45.57 & 55.04 \\
\hline
\end{tabular}

TABlE 5. Estimated C155 runtimes in days.

\begin{tabular}{|r|r|r|r|r|r|r|r|r|r|}
\hline$t$ & $r$ & $F(t)$ & $L P$ & $E(t)$ & $E_{L P}(t)$ & $T(t)$ & $T$ & $L A$ & total \\
\hline
\end{tabular}

situation, with the relation generation being done in parallel on 256 processors, we should use the variation of large primes that considers the set of large prime divisors to include those divisors having norms with degree equal to $t+1$ in all four curves. It also appears that we should set $r=1$ for all four curves. See Table 6 for a summary of what we used for input for random walks. The large prime bound is the degree of the norms of divisors that we use in our set of large prime divisors. We also list in Table 7 the settings from [25] for reference and comparison.

TABLE 6. Random walk parameters for the Enge-Gaudry algorithm.

\begin{tabular}{|r|l|l|l|l|}
\hline Curve & C62 & C93 & C124 & C155 \\
\hline Factor Base Bound $t$ & 6 & 5 & 4 & 4 \\
Parameter $r$ & 1 & 1 & 1 & 1 \\
Large Prime Bound & 7 & 6 & 5 & 5 \\
Estimated Time & 12.65 Seconds & 14.72 Minutes & 24.24 Hours & 27.56 Days \\
\hline
\end{tabular}


TABLE 7. Random walk parameters from [25] for the Enge-Gaudry algorithm.

\begin{tabular}{|r|l|l|l|l|}
\hline Curve & C62 & C93 & C124 & C155 \\
\hline Factor Base Bound $t$ & 7 & 5 & 5 & 4 \\
Parameter $r$ & 1 & 1 & 1 & 1 \\
Estimated Time & 23.48 Seconds & 20.77 Minutes & 50.59 Hours & 35.65 Days \\
\hline
\end{tabular}

TABLE 8. Parameters for sieve-based algorithm.

\begin{tabular}{|r|l|l|l|l|}
\hline Curve & C62 & C93 & C124 & C155 \\
\hline Factor Base Bound $t$ & 6 & 5 & 4 & 4 \\
Parameter $r$ & 1 & 1 & 1 & 1 \\
Large Prime Bound & 7 & 6 & 5 & 5 \\
Divisors used $j$ & 5 & 7 & 9 & 9 \\
Radius Degree $M$ & 4 & 3 & 2 & 2 \\
Tolerance Value $Y$ & 19 & 21 & 25 & 25 \\
\hline
\end{tabular}

We used the settings in Table [6 as a starting point for selecting the sieve parameters. The remaining parameters were generated by starting with the strategy described in Section 4.2. The final parameters selected are listed in Table 8.

Numerical results. We have used our implementation to solve discrete logarithm problems in C62, C93, and C124 using both the Enge-Gaudry algorithm and our sieve-based adaptation of Vollmer's algorithm with self-initialized sieving and parameters as selected above. Tables 9 to 12 list the results for $\mathrm{C} 62$ to $\mathrm{C} 155$, respectively. We list estimated and actual runtimes and statistics using the strategy and optimal parameters from [25] (labeled as "JMS"), our optimized version incorporating large primes, and our sieve-based version of Vollmer's algorithm. For each algorithm, we give the following data:

- Initialization Time: time required to compute the factor base

- Factor Base Size: number of prime divisors in the factor base

- Total Relations: number of full relations found, including those computed from partial relations (sum of "Full Relations" and "Unique Intersections")

- Full Relations: number of full relations found directly, i.e., that were not constructed from partial relations

- Almost Smooth Relations: number of partial relations found

- Intersections and Unique Intersections: number of intersections of partial relations leading to full relations, and the number of these that were unique

- Sieve Polynomials: number of sieve polynomials used in the sieve-based algorithm

- Total Divisors Tested: number of random walk steps, or, for the sievebased algorithm, the number of sieve polynomials multiplied by the size of the sieve radius $q^{M+1}$.

- Total Search Time and Real Search Time: the total amount of CPU time spent searching for relations, and the real time spent (total time divided by the number of CPUs used)

- Total Iterations: number of attempts required before the linear system generated had a solution (and the discrete logarithm was found) 
TABLE 9. C62 results

\begin{tabular}{|c|c|c|c|c|c|}
\hline & $\begin{array}{l}\text { JMS EG } \\
\text { Estimate }\end{array}$ & $\begin{array}{l}\text { JMS EG } \\
\text { Result }\end{array}$ & $\begin{array}{l}\text { Opt. EG } \\
\text { Estimate }\end{array}$ & $\begin{array}{l}\text { Opt. EG } \\
\text { Result }\end{array}$ & $\begin{array}{l}\text { Sieving } \\
\text { Result }\end{array}$ \\
\hline Initialization Time & $6.18 \mathrm{~s}$ & $6.28 \mathrm{~s}$ & $2.96 \mathrm{~s}$ & $2.73 \mathrm{~s}$ & $0.76 \mathrm{~s}$ \\
\hline Total Relations & 1649 & 1649 & 479 & 479 & 489 \\
\hline Full Relations & 1649 & 1649 & 310 & 302 & 321 \\
\hline $\begin{array}{r}\text { Almost } \\
\text { Smooth Relations }\end{array}$ & - & - & 770 & 780 & 986 \\
\hline Intersections & - & - & 169 & 198 & 180 \\
\hline Unique Intersections & - & - & 169 & 198 & 180 \\
\hline Sieve Polynomials & - & - & - & - & 618361 \\
\hline Total Divisors Tested & 694997 & 590394 & 809562 & 640471 & 633201664 \\
\hline Total Search Time & $32.31 \mathrm{~m}$ & $27 \mathrm{~m} \mathrm{25.31s}$ & $37.44 \mathrm{~m}$ & $27 \mathrm{~m} 51.86 \mathrm{~s}$ & $21 \mathrm{~m} 40.78 \mathrm{~s}$ \\
\hline Real Search Time & $7.57 \mathrm{~s}$ & $6.42 \mathrm{~s}$ & $8.78 \mathrm{~s}$ & $6.53 \mathrm{~s}$ & $8.21 \mathrm{~s}$ \\
\hline Total Iterations & - & 1 & - & 1 & 3 \\
\hline $\begin{array}{r}\text { Special } \\
\text { Divisors Checked }\end{array}$ & - & - & - & - & 1081344 \\
\hline Special Rels Time & - & - & - & - & $0.99 \mathrm{~s}$ \\
\hline Linear Algebra Time & $9.73 \mathrm{~s}$ & $34.45 \mathrm{~s}$ & $0.92 \mathrm{~s}$ & $2.85 \mathrm{~s}$ & $13.03 \mathrm{~s}$ \\
\hline Total Time & $32.57 \mathrm{~m}$ & $28 \mathrm{~m} 6.66 \mathrm{~s}$ & $37.51 \mathrm{~m}$ & $27 \mathrm{~m} 57.91 \mathrm{~s}$ & $21 \mathrm{~m} 56.45 \mathrm{~s}$ \\
\hline Real Total Time & $23.48 \mathrm{~s}$ & $47.77 \mathrm{~s}$ & $12.65 \mathrm{~s}$ & $12.58 \mathrm{~s}$ & $22.90 \mathrm{~s}$ \\
\hline
\end{tabular}

- Special Divisors Checked and Special Rels Time: for Vollmer's algorithm, the total number of divisors tested before factorizations of the input divisors $D_{1}$ and $D_{2}$ were found, and the time required to perform these factorizations

- Linear Algebra Time: total time spent solving linear systems

- Total Time and Real Time: total CPU time spent on the algorithm; total real time spent (i.e., accounting for parallelism)

First notice that the linear algebra times were significantly underestimated in all cases. Using more accurate estimates might result in different choices for $t$ and $r$ as we attempt to choose settings that result in the lowest runtime. However, in most cases it is the search for smooth relations that dominated the computation time, especially when considering the total times as opposed to the real time required with 256 processors.

For the C62 case (Table 9) we notice that significantly fewer divisors were tested than we expected in both the version of Enge-Gaudry from [25] and our optimized version. One possible cause of this is when a large number of smooth or almost smooth relations are found early in random walks. This, spread over the 256 random walks occurring, could explain this difference. Further evidence for this idea was provided when we ran the same test using a smaller number of processors. Tests using both 10 processors and 1 processor resulted in the number of divisors tested to be much closer to the estimates. We also point out that in our optimized version of Enge-Gaudry we expected $T(t) / E_{L P}(t)=809562 / 1051 \approx 770$ almost smooth relations to be found, and this is almost exactly what we did find. Comparing our optimal results to the results produced using the version from 25, we see that the search times are almost the same, but due to the larger factor base size recommended by JMS, the linear algebra time is significantly larger. Finally, we note that the time spent searching for relations was improved significantly using sieving. Unfortunately, the first relation matrix did not yield a solution to the 
TABLE 10. C93 results

\begin{tabular}{|c|c|c|c|c|c|}
\hline & $\begin{array}{l}\text { JMS EG } \\
\text { Estimate }\end{array}$ & $\begin{array}{l}\text { JMS EG } \\
\text { Result }\end{array}$ & $\begin{array}{l}\text { Opt. EG } \\
\text { Estimate }\end{array}$ & $\begin{array}{l}\text { Opt. EG } \\
\text { Result }\end{array}$ & $\begin{array}{l}\text { Sieving } \\
\text { Result }\end{array}$ \\
\hline $\begin{array}{r}\text { Initialization } \\
\text { Time }\end{array}$ & $9.67 \mathrm{~s}$ & $9.76 \mathrm{~s}$ & $9.67 \mathrm{~s}$ & $8.70 \mathrm{~s}$ & $4.55 \mathrm{~s}$ \\
\hline $\begin{array}{r}\text { Total } \\
\text { Relations }\end{array}$ & 3877 & 3877 & 3877 & 3877 & 3892 \\
\hline $\begin{array}{r}\text { Full } \\
\text { Relations }\end{array}$ & 3877 & 3877 & 2504 & 2480 & 2547 \\
\hline $\begin{array}{r}\text { Almost Smooth } \\
\text { Relations }\end{array}$ & - & - & 9475 & 9546 & 13246 \\
\hline Intersections & - & - & 1373 & 1834 & 5271 \\
\hline $\begin{array}{r}\text { Unique } \\
\text { Intersections }\end{array}$ & - & - & 1373 & 1834 & 1496 \\
\hline $\begin{array}{r}\text { Sieve } \\
\text { Polynomials }\end{array}$ & - & - & - & - & 8889395 \\
\hline $\begin{array}{r}\text { Total Divisors } \\
\text { Tested }\end{array}$ & 111146195 & 101080503 & 71792103 & 70951503 & 36410961920 \\
\hline $\begin{array}{r}\text { Total Search } \\
\text { Time }\end{array}$ & $3.42 \mathrm{~d}$ & $\begin{array}{c}3 \mathrm{~d} 4 \mathrm{~h} \\
2 \mathrm{~m} 9.10 \mathrm{~s}\end{array}$ & $2.32 \mathrm{~d}$ & $\begin{array}{c}2 \mathrm{~d} 5 \mathrm{~h} \\
41 \mathrm{~m} 16.87 \mathrm{~s}\end{array}$ & $\begin{array}{c}11 \mathrm{~h} 36 \mathrm{~m} \\
43.94 \mathrm{~s}\end{array}$ \\
\hline $\begin{array}{r}\text { Real Search } \\
\text { Time }\end{array}$ & $19.26 \mathrm{~m}$ & $17 \mathrm{~m} 49.25 \mathrm{~s}$ & $13.03 \mathrm{~m}$ & $12 \mathrm{~m} \mathrm{34.98s}$ & $3 \mathrm{~m} 13.40 \mathrm{~s}$ \\
\hline $\begin{array}{r}\text { Total } \\
\text { Iterations }\end{array}$ & - & 1 & - & 1 & 3 \\
\hline $\begin{array}{r}\text { Special Divisors } \\
\text { Checked }\end{array}$ & - & - & - & - & 11501568 \\
\hline $\begin{array}{r}\text { Special Rels } \\
\text { Time } \\
\end{array}$ & - & - & - & - & $7.92 \mathrm{~s}$ \\
\hline $\begin{array}{r}\text { Linear Algebra } \\
\text { Time }\end{array}$ & $1.35 \mathrm{~m}$ & $6 \mathrm{~m} 5.73 \mathrm{~s}$ & $1.53 \mathrm{~m}$ & $4 \mathrm{~m} 57.28 \mathrm{~s}$ & $22 \mathrm{~m} 7.37 \mathrm{~s}$ \\
\hline Total Time & $3.43 \mathrm{~d}$ & $\begin{array}{c}3 \mathrm{~d} 4 \mathrm{~h} \\
8 \mathrm{~m} 26.11 \mathrm{~s}\end{array}$ & $2.32 \mathrm{~d}$ & $\begin{array}{c}2 \mathrm{~d} 5 \mathrm{~h} \\
46 \mathrm{~m} 28.02 \mathrm{~s}\end{array}$ & $\begin{array}{c}11 \mathrm{~h} 58 \mathrm{~m} \\
59.23 \mathrm{~s}\end{array}$ \\
\hline Real Total Time & $20.77 \mathrm{~m}$ & $24 \mathrm{~m} 6.26 \mathrm{~s}$ & $14.72 \mathrm{~m}$ & $17 \mathrm{~m} \mathrm{46.13s}$ & $25 \mathrm{~m} \mathrm{20.80s}$ \\
\hline
\end{tabular}

HCDLP, and in fact the linear algebra had to be repeated three times, negating the impact of the speed-up in relation generation. Even so, the total time spent on all processors was the least when using Vollmer's algorithm, but the fact that the relation generalization was distributed over 256 processors meant that the real time was not.

For C93 (Table 10) we see that the number of divisors tested in both the JMS and optimal results are much closer to that which we expected. This time the same factor base size was recommended for the version from 25 and our optimized version. Here we see that using large primes does reduce the amount of time needed in the search. Again, when comparing the results to the sieve-based algorithm, we see that while relation generation with sieving is significantly faster, taking around a quarter of the time, once again the linear algebra took three iterations. This significantly increases the amount of time this test took to run on the cluster, as the linear algebra implementation we used is not parallelized. If adjusting the algorithm to produce the extra relations results in a reduction of the number of linear algebra iterations, then this would result in the sieve-based method being significantly better here. We also note that while there were a significant number of almost smooth relations and intersections in the sieve-based method, a large number of the 
TABLE 11. C124 results

\begin{tabular}{|c|c|c|c|c|c|}
\hline & $\begin{array}{l}\text { JMS EG } \\
\text { Estimate }\end{array}$ & $\begin{array}{l}\text { JMS EG } \\
\text { Result }\end{array}$ & $\begin{array}{l}\text { Opt. EG } \\
\text { Estimate }\end{array}$ & $\begin{array}{l}\text { Opt. EG } \\
\text { Result }\end{array}$ & $\begin{array}{l}\text { Sieving } \\
\text { Result }\end{array}$ \\
\hline $\begin{array}{r}\text { Initialization } \\
\text { Time }\end{array}$ & $5.56 \mathrm{~m}$ & $5 \mathrm{~m} 44.75 \mathrm{~s}$ & $17.64 \mathrm{~s}$ & $15.59 \mathrm{~s}$ & $10.44 \mathrm{~s}$ \\
\hline Total Relations & 113733 & 113733 & 8879 & 8879 & 8870 \\
\hline Full Relations & 113733 & 113733 & 5154 & 5176 & 5084 \\
\hline $\begin{array}{r}\text { Almost } \\
\text { Smooth Relations } \\
\end{array}$ & - & - & 34232 & 34106 & 34520 \\
\hline Intersections & - & - & 3725 & 4953 & 4510 \\
\hline $\begin{array}{r}\text { Unique } \\
\text { Intersections }\end{array}$ & - & - & 3725 & 4953 & 4510 \\
\hline $\begin{array}{r}\text { Sieve } \\
\text { Polynomials }\end{array}$ & - & - & - & - & 1581411077 \\
\hline $\begin{array}{r}\text { Total Divisors } \\
\text { Tested }\end{array}$ & 2942900860 & 2931742632 & 7721296965 & 7557879515 & 6477459771392 \\
\hline $\begin{array}{r}\text { Total Search } \\
\text { Time }\end{array}$ & $110.32 \mathrm{~d}$ & $\begin{array}{l}115 \mathrm{~d} 5 \mathrm{~h} \\
56 \mathrm{~m} 15.77 \mathrm{~s}\end{array}$ & $255.59 \mathrm{~d}$ & $\begin{array}{l}243 \mathrm{~d} 23 \mathrm{~h} \\
42 \mathrm{~m} 45.22 \mathrm{~s}\end{array}$ & $\begin{array}{l}81 \mathrm{~d} 6 \mathrm{~h} \\
59 \mathrm{~m} 28.12 \mathrm{~s}\end{array}$ \\
\hline $\begin{array}{r}\text { Real Search } \\
\text { Time }\end{array}$ & $10.34 \mathrm{~h}$ & $\begin{array}{l}10 \mathrm{~h} \\
48 \mathrm{~m} 15.99 \mathrm{~s}\end{array}$ & $23.96 \mathrm{~h}$ & $\begin{array}{l}22 \mathrm{~h} \\
52 \mathrm{~m} 25.95 \mathrm{~s}\end{array}$ & $\begin{array}{l}7 \mathrm{~h} \\
37 \mathrm{~m} 14.86 \mathrm{~s}\end{array}$ \\
\hline $\begin{array}{r}\text { Total } \\
\text { Iterations }\end{array}$ & - & 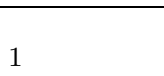 & - & 1 & 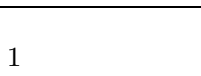 \\
\hline $\begin{array}{r}\text { Special Divisors } \\
\text { Checked }\end{array}$ & - & - & - & - & 143921152 \\
\hline $\begin{array}{r}\text { Special Rels } \\
\text { Time }\end{array}$ & - & - & - & - & $3 \mathrm{~m} \mathrm{34.67s}$ \\
\hline $\begin{array}{r}\text { Linear Algebra } \\
\text { Time }\end{array}$ & $40.15 \mathrm{~h}$ & & $0.28 \mathrm{~h}$ & $37 \mathrm{~m} 43.56 \mathrm{~s}$ & 1h $44.90 \mathrm{~s}$ \\
\hline Total Time & $112 \mathrm{~d}$ & & $255.60 \mathrm{~d}$ & $\begin{array}{l}244 \mathrm{~d} \\
21 \mathrm{~m} 2.53 \mathrm{~s}\end{array}$ & $\begin{array}{l}81 \mathrm{~d} 8 \mathrm{~h} \\
3 \mathrm{~m} 47.69 \mathrm{~s}\end{array}$ \\
\hline Real Total Time & $50.59 \mathrm{~h}$ & & $24.24 \mathrm{~h}$ & $\begin{array}{l}23 \mathrm{~h} \\
30 \mathrm{~m} 43.26 \mathrm{~s}\end{array}$ & $\begin{array}{l}8 \mathrm{~h} \\
42 \mathrm{~m} 3.49 \mathrm{~s}\end{array}$ \\
\hline
\end{tabular}

relations formed by the intersections were discarded as being duplicate relations. This consistently happened, and we can offer no explanation for this behavior.

We now consider the results for C124 in Table 11. Again our results are quite close to the expected values. Relation generation takes significantly longer with our optimized version of the Enge-Gaudry algorithm than with the version in 25. However, we note that due to the larger factor base required in [25], we expect the linear algebra to take significantly longer in that case. Since the difference in the search time can be spread out over a parallel system but the linear algebra cannot, our settings should be better, and increasing the number of processors would only improve that situation. However the most dramatic result that we see is that from the sieve-based algorithm. Here the search time is less than the time using [25, and additionally, while the linear algebra is worse than that in our optimal EngeGaudry case, it is significantly better than that using the settings from [25], giving us a very significant improvement.

Finally we examine the results for C155 in Table 12. Once again we see that the use of sieving results in a much faster search for relations when compared to the random walk estimates we have computed. In this case, the search takes approximately a quarter of the time that we expect it to take with the Enge-Gaudry algorithm. As mentioned before, the linear algebra estimates are very wrong and 
TABLE 12. C155 results

\begin{tabular}{|c|c|c|c|}
\hline & $\begin{array}{l}\text { JMS EG } \\
\text { Estimate }\end{array}$ & $\begin{array}{l}\text { Opt. EG } \\
\text { Estimate }\end{array}$ & $\begin{array}{l}\text { Sieving } \\
\text { Result }\end{array}$ \\
\hline $\begin{array}{r}\text { Initialization } \\
\text { Time }\end{array}$ & $5.95 \mathrm{~m}$ & $5.95 \mathrm{~m}$ & $6 \mathrm{~m} 4.87 \mathrm{~s}$ \\
\hline Total Relations & 136533 & 136533 & 136533 \\
\hline Full Relations & 136533 & 96662 & 99226 \\
\hline Partial Relations & - & 633522 & 612780 \\
\hline Intersections & - & 39871 & 51660 \\
\hline $\begin{array}{r}\text { Unique } \\
\text { Intersections }\end{array}$ & - & 39871 & 51660 \\
\hline $\begin{array}{r}\text { Sieve } \\
\text { Polynomials }\end{array}$ & - & - & 3036124745 \\
\hline $\begin{array}{r}\text { Total Divisors } \\
\text { Tested }\end{array}$ & 188193560300 & 133236952000 & 99484699519415 \\
\hline $\begin{array}{r}\text { Total Search } \\
\text { Time }\end{array}$ & $8492.67 \mathrm{~d}$ & $6338.01 \mathrm{~d}$ & 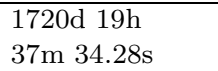 \\
\hline $\begin{array}{r}\text { Real Search } \\
\text { Time }\end{array}$ & $33.17 \mathrm{~d}$ & $24.76 \mathrm{~d}$ & $\begin{array}{l}\text { 6d 21h } \\
15 \mathrm{~m} \mathrm{44.92s}\end{array}$ \\
\hline $\begin{array}{r}\text { Total } \\
\text { Iterations }\end{array}$ & - & - & 1 \\
\hline $\begin{array}{r}\text { Special Divisors } \\
\text { Checked }\end{array}$ & - & - & 177531606 \\
\hline $\begin{array}{r}\text { Special Rels } \\
\text { Time }\end{array}$ & - & - & $6 \mathrm{~m} 35.15 \mathrm{~s}$ \\
\hline $\begin{array}{r}\text { Linear Algebra } \\
\text { Time }\end{array}$ & $2.47 \mathrm{~d}$ & $2.80 \mathrm{~d}$ & $\begin{array}{l}14 \mathrm{~d} 5 \mathrm{~h} \\
51 \mathrm{~m} 26.46 \mathrm{~s}\end{array}$ \\
\hline Total Time & $8495.14 d$ & $6340.81 \mathrm{~d}$ & $\begin{array}{l}1735 \mathrm{~d} \mathrm{1h} \\
30 \mathrm{~m} 6.81 \mathrm{~s}\end{array}$ \\
\hline Real Total Time & $35.65 \mathrm{~d}$ & $27.56 \mathrm{~d}$ & $\begin{array}{l}21 \mathrm{~d} 7 \mathrm{~h} \\
39 \mathrm{~m} 17.12 \mathrm{~s}\end{array}$ \\
\hline
\end{tabular}

require further investigation. Because the linear algebra algorithm used depends both on the size of the matrix and the number of non-zero entries in the matrix, we see the large jump in linear algebra time from the C124 example to the C155 example. This result makes computing linear algebra estimates for the C155 case difficult. In any case, because our parameter search suggests that we would be using the same size factor base for both the Enge-Gaudry algorithm and the sievebased algorithm, we would expect the linear algebra time to be close to the same for both. Correcting our optimal Enge-Gaudry estimates with this observation, we see that the sieve-based algorithm takes little more than half the time we expect the Enge-Gaudry algorithm to take in its best case

Our results show that using sieving to compute relations can be much faster than using random walks. In conjunction with our adaptation of Vollmer's algorithm for solving the discrete logarithm problem, we have a more efficient algorithm for computing discrete logarithms in divisor class groups of hyperelliptic curves defined over even characteristic finite fields.

\section{FURTHER WORK}

There are several potential improvements and extensions possible with this work. A number of these, most of which are already work in progress, are listed below. 
Although the introduction of large primes and sieving does result in significant performance improvements in practice as opposed to the random walks strategy, there is still further room for improvement. For example, the parameter selection for the random walks strategy is currently based on an estimate of the factor base size. The analysis could be refined by computing the exact factor base size ahead of time and using it instead. Besides possibly yielding further performance gains, this may help explain the differences between our expected and observed running times. In addition, the empirical analysis used to find optimal parameters for our improved versions of the random walks strategy can likely be extended to our sievebased version of Vollmer's algorithm. While we do some analysis in this case, the final parameter selection is currently done empirically. A careful analysis may yield optimal parameter choices without the experimental trials.

Further algorithmic improvements are also foreseeable. A double large prime variant, again adapted from integer factorization, is described in 16] for solving the HCDLP in the low-genus case. It should be equally applicable to high-genus curves; work on adapting our optimal parameter selection strategy is in progress. We used the bound $t+1$ for large primes; one could also investigate whether using $t+2$ or higher results in further improvements. Improved sieving and smoothness testing methods, possibly representing the sieve array as a multidimensional array indexed by the coefficients of the index polynomials, as well as Bernstein's batch smoothness test [4, may also yield improvements in performance. An analysis of these methods should help quantify the effectiveness of these potential improvements.

The examples we chose for this paper are interesting in that they shed some light on the feasibility of the Weil descent attack on the ECDLP. However, it would certainly be interesting the explore the utility of our algorithms in additional settings, including lower-genus curves and curves defined over odd characteristic finite fields. An investigation into the performance of the algorithm as $g$ and $q$ vary would be useful in finding the limits of our methods. A careful complexity analysis of the algorithm, with and without sieving, might lead to asymptotic improvements as well.

It is well known 10,41 that the index calculus methods described in this paper can be adapted easily to compute the order and structure of the Jacobian $J_{C}(k)$. Roughly, the same relation generation strategies can be employed, including the improvements described in this paper, resulting in a relation matrix $A$ whose columns correspond to principal divisors. Computing the determinant of the lattice generated by the columns of $A$ and the Smith normal form of $A$ yields $\# J_{C}(k)$ and the elementary divisors of $J_{C}(k)$, respectively. The required linear algebra is significantly harder than solving $A \vec{x}=\vec{b}\left(\bmod \# J_{C}(k)\right)$, as is required for computing the HCDLP. It would be interesting to extend our empirical analysis to this case, taking into account the increased complexity of the linear algebra, and see how efficiently we can compute these invariants with our improved algorithms.

Finally, in [35, Müller, Stein, and Thiel present index calculus algorithms for computing the regulator and solving the infrastructure discrete logarithm problem in a real quadratic function field. Recent work by Hammell [20, 21] shows that the sieving approach discussed here gives similar results in this setting. However, attempts to apply sieving to real quadratic function fields of odd characteristic were not as successful. More work is required to determine whether sieving can be made more efficient in this setting and in the low-genus case. 


\section{REFERENCES}

[1] W. R. Alford and Carl Pomerance, Implementing the self-initializing quadratic sieve on a distributed network, Number-theoretic and algebraic methods in computer science (Moscow, 1993), World Sci. Publ., River Edge, NJ, 1995, pp. 163-174. MR1377748 (96k:11152)

[2] ATLAS, Automatically tuned linear algebra software, Software, 2007, See http:// math-atlas.sourceforge.net/.

[3] Eric Bach and Jeffrey Shallit, Algorithmic number theory. Vol. 1, Foundations of Computing Series, MIT Press, Cambridge, MA, 1996. Efficient algorithms. MR1406794 (97e:11157)

[4] D. Bernstein, How to find small factors of integers, to appear in Math. Comp.

[5] David G. Cantor, Computing in the Jacobian of a hyperelliptic curve, Math. Comp. 48 (1987), no. 177, 95-101, DOI 10.2307/2007876. MR866101 (88f:11118)

[6] David G. Cantor and Hans Zassenhaus, A new algorithm for factoring polynomials over finite fields, Math. Comp. 36 (1981), no. 154, 587-592, DOI 10.2307/2007663. MR606517 (82e:12020)

[7] Henri Cohen, Gerhard Frey, Roberto Avanzi, Christophe Doche, Tanja Lange, Kim Nguyen, and Frederik Vercauteren (eds.), Handbook of elliptic and hyperelliptic curve cryptography, Discrete Mathematics and its Applications (Boca Raton), Chapman \& Hall/CRC, Boca Raton, FL, 2006. MR2162716 (2007f:14020)

[8] S. Contini, Factoring integers with the self-initializing quadratic sieve, Master's thesis, University of Georgia, Athens, Georgia, 1997.

[9] Richard Crandall and Carl Pomerance, Prime numbers, a computational perspective, Springer-Verlag, New York, 2001. MR.1821158 (2002a:11007)

[10] Andreas Enge and Pierrick Gaudry, A general framework for subexponential discrete logarithm algorithms, Acta Arith. 102 (2002), no. 1, 83-103, DOI 10.4064/aa102-1-6. MR1884958 (2002k:11225)

[11] Ralf Flassenberg and Sachar Paulus, Sieving in function fields, Experiment. Math. 8 (1999), no. 4, 339-349. MR1737230 (2000j:11179)

[12] G. Frey, How to disguise an elliptic curve (Weil descent), Talk at ECC '98, Waterloo, 1998. Slides available from http://www.cacr.math.uwaterloo.ca/conferences/1998/ecc98/ slides.html

[13] Gerhard Frey, Applications of arithmetical geometry to cryptographic constructions, Finite fields and applications (Augsburg, 1999), Springer, Berlin, 2001, pp. 128-161. MR.1849086 (2002h:11136)

[14] Pierrick Gaudry, An algorithm for solving the discrete log problem on hyperelliptic curves, Advances in cryptology -EUROCRYPT 2000 (Bruges), Lecture Notes in Comput. Sci., vol. 1807, Springer, Berlin, 2000, pp. 19-34, DOI 10.1007/3-540-45539-6_2. MR.1772021

[15] P. Gaudry, F. Hess, and N. P. Smart, Constructive and destructive facets of Weil descent on elliptic curves, J. Cryptology 15 (2002), no. 1, 19-46, DOI 10.1007/s00145-001-0011-x. MR.1880933 (2003b:14032)

[16] P. Gaudry, E. Thomé, N. Thériault, and C. Diem, A double large prime variation for small genus hyperelliptic index calculus, Math. Comp. 76 (2007), no. 257, 475-492 (electronic), DOI 10.1090/S0025-5718-06-01900-4. MR2261032 (2007j:11174)

[17] Pierrick Gaudry, An algorithm for solving the discrete log problem on hyperelliptic curves, Advances in cryptology_EUROCRYPT 2000 (Bruges), Lecture Notes in Comput. Sci., vol. 1807, Springer, Berlin, 2000, pp. 19-34, DOI 10.1007/3-540-45539-6_2. MR.1772021

[18] GCC, GCC, the GNU compiler collection, Software, 2007, see http://gcc.gnu.org/.

[19] GMP, The GNU multiple precision bignum library, Software, 2007, see http://gmplib.org/.

[20] J. F. Hammell, Index calculus in the infrastructure of real quadratic function fields, Master's thesis, University of Calgary, Canada, 2008.

[21] J. F. Hammell and M. J. Jacobson, Jr., Index-calculus algorithms in real quadratic function fields, In preparation, 2011.

[22] Michael J. Jacobson Jr., Applying sieving to the computation of quadratic class groups, Math. Comp. 68 (1999), no. 226, 859-867, DOI 10.1090/S0025-5718-99-01003-0. MR.1604324 (99i:11120)

[23] Michael J. Jacobson Jr., Subexponential class group computation in quadratic orders, Ph.D. thesis, Technische Universität Darmstadt, Darmstadt, Germany, 1999. 
[24] Michael J. Jacobson Jr., Computing discrete logarithms in quadratic orders, J. Cryptology 13 (2000), no. 4, 473-492, DOI 10.1007/s001450010013. MR1788516 (2003b:94046)

[25] Michael Jacobson, Alfred Menezes, and Andreas Stein, Solving elliptic curve discrete logarithm problems using Weil descent, J. Ramanujan Math. Soc. 16 (2001), no. 3, 231-260. MR:1863606(2002h:14039)

[26] M. J. Jacobson Jr., R. Scheidler, and A. Stein, Fast arithmetic on hyperelliptic curves via continued fraction expansions, Advances in coding theory and cryptography, Ser. Coding Theory Cryptol., vol. 3, World Sci. Publ., Hackensack, NJ, 2007, pp. 200-243, DOI 10.1142/9789812772022_0013. MR2454114 (2010a:14054)

[27] Neal Koblitz, Algebraic aspects of cryptography, Algorithms and Computation in Mathematics, vol. 3, Springer-Verlag, Berlin, 1998. With an appendix by Alfred J. Menezes, Yi-Hong $\mathrm{Wu}$ and Robert J. Zuccherato. MR1610535(2000a:94012)

[28] Neal Koblitz, Elliptic curve cryptosystems, Math. Comp. 48 (1987), no. 177, 203-209, DOI 10.2307/2007884. MR866109(88b:94017)

[29] Neal Koblitz, Hyperelliptic cryptosystems, J. Cryptology 1 (1989), no. 3, 139-150, DOI 10.1007/BF02252872. MR1007215 (90k:11165)

[30] H. W. Lenstra Jr., The number field sieve: an annotated bibliography, The development of the number field sieve, Lecture Notes in Math., vol. 1554, Springer, Berlin, 1993, pp. 1-3, DOI 10.1007/BFb0091535. MR.1321217

[31] LinBox, Project LinBox: Exact computational linear algebra, Software, 2007, see http: //www . linalg.org/.

[32] Markus Maurer, Alfred Menezes, and Edlyn Teske, Analysis of the GHS Weil descent attack on the ECDLP over characteristic two finite fields of composite degree, LMS J. Comput. Math. 5 (2002), 127-174, DOI 10.1007/3-540-45311-3_19. MR1942257(2003k:94034)

[33] MPI, Message passing interface, Software, 2007, see http://www-unix.mcs.anl.gov/mpi/.

[34] Victor S. Miller, Use of elliptic curves in cryptography, Advances in cryptology-CRYPTO '85 (Santa Barbara, Calif., 1985), Lecture Notes in Comput. Sci., vol. 218, Springer, Berlin, 1986, pp. 417-426, DOI 10.1007/3-540-39799-X_31. MR851432 (88b:68040)

[35] Volker Müller, Andreas Stein, and Christoph Thiel, Computing discrete logarithms in real quadratic congruence function fields of large genus, Math. Comp. 68 (1999), no. 226, 807-822, DOI 10.1090/S0025-5718-99-01040-6. MR.1620235 (99i:11119)

[36] Carl Pomerance, The quadratic sieve factoring algorithm, Advances in cryptology (Paris, 1984), Lecture Notes in Comput. Sci., vol. 209, Springer, Berlin, 1985, pp. 169-182, DOI 10.1007/3-540-39757-4_17. MR825590(87d:11098)

[37] V. Shoup, NTL: A library for doing number theory, http://www.shoup.net, 2008.

[38] Edlyn Teske, Speeding up Pollard's rho method for computing discrete logarithms, Algorithmic number theory (Portland, OR, 1998), Lecture Notes in Comput. Sci., vol. 1423, Springer, Berlin, 1998, pp. 541-554, DOI 10.1007/BFb0054891. MR.1726100 (2000j:11199)

[39] Nicolas Thériault, Index calculus attack for hyperelliptic curves of small genus, Advances in cryptology-ASIACRYPT 2003, Lecture Notes in Comput. Sci., vol. 2894, Springer, Berlin, 2003, pp. 75-92, DOI 10.1007/978-3-540-40061-5_5. MR2093253 (2006d:94068)

[40] M. D. Velichka, Improvements to index calculus algorithms for solving the hyperelliptic curve discrete logarithm problem over characteristic two finite fields, Master's thesis, University of Calgary, Canada, 2008.

[41] Ulrich Vollmer, Asymptotically fast discrete logarithms in quadratic number fields, Algorithmic number theory (Leiden, 2000), Lecture Notes in Comput. Sci., vol. 1838, Springer, Berlin, 2000, pp. 581-594, DOI 10.1007/10722028_39. MR1850635(2003b:11135)

[42] Joachim von zur Gathen and Jürgen Gerhard, Modern computer algebra, Cambridge University Press, New York, 1999. MR1689167 (2000j:68205) 
Department of Computer Science, University of Calgary, 2500 University Drive NW, Calgary, Alberta, Canada T2N 1N4

E-mail address: markvelichka@gmail.com

Department of Computer Science, University of Calgary, 2500 University Drive NW, Calgary, Alberta, Canada T2N 1N4

E-mail address: jacobs@cpsc.ucalgary.ca

Institut für Mathematik, Carl-von-Ossietzky Universität Oldenburg, D-26111 OldENBURG, GERMANY

E-mail address: andreas.stein1@uni-oldenburg.de 